\title{
BAYESIAN HIGH-REDSHIFT QUASAR CLASSIFICATION FROM OPTICAL AND MID-IR PHOTOMETRY
}

\author{
Gordon T. Richards ${ }^{1,2}$, Adam D. Myers ${ }^{2,3}$, Christina M. Peters ${ }^{1}$, Coleman M. KrawczyK ${ }^{1}$, \\ Greg Chase ${ }^{1}$, Nicholas P. Ross ${ }^{1}$, XiaOhui Fan ${ }^{4}$, Linhua Jiang ${ }^{5}$, Mark Lacy ${ }^{6}$, Ian D. McGreer ${ }^{4}$, \\ Jonathan R. TRUMP ${ }^{7}$, AND RyAN N. RIEGEL ${ }^{8}$ \\ ${ }^{1}$ Department of Physics, Drexel University, 3141 Chestnut Street, Philadelphia, PA 19104, USA \\ ${ }_{2}^{2}$ Max Planck Institut für Astronomie, Königstuhl 17, Heidelberg, D-69117, Germany \\ ${ }^{3}$ Department of Physics and Astronomy, University of Wyoming, Laramie, WY 82071, USA \\ ${ }^{4}$ Steward Observatory, University of Arizona, Tucson, AZ 85721, USA \\ ${ }^{5}$ Kavli Institute for Astronomy and Astrophysics, Peking University, Beijing 100871, P.R. China \\ ${ }^{6}$ National Radio Astronomy Observatory, 520 Edgemont Road, Charlottesville, VA 22903, USA \\ ${ }^{7}$ Department of Astronomy and Astrophysics, 525 Davey Lab, The Pennsylvania State University, University Park, PA 16802, USA \\ ${ }^{8}$ Skytree, Inc., 1731 Technology Drive, Suite 700, San Jose, CA 95110, USA \\ Received 2015 February 10; accepted 2015 July 24; published 2015 August 24
}

\begin{abstract}
We identify 885,503 type 1 quasar candidates to $i \lesssim 22$ using the combination of optical and mid-IR photometry. Optical photometry is taken from the Sloan Digital Sky Survey-III: Baryon Oscillation Spectroscopic Survey (SDSS-III/BOSS), while mid-IR photometry comes from a combination of data from the Wide-field Infrared Survey Explorer (WISE) "AllWISE" data release and several large-area Spitzer Space Telescope fields. Selection is based on a Bayesian kernel density algorithm with a training sample of 157,701 spectroscopically confirmed type 1 quasars with both optical and mid-IR data. Of the quasar candidates, 733,713 lack spectroscopic confirmation (and 305,623 are objects that we have not previously classified as photometric quasar candidates). These candidates include 7874 objects targeted as high-probability potential quasars with $3.5<z<5$ (of which 6779 are new photometric candidates). Our algorithm is more complete to $z>3.5$ than the traditional mid-IR selection "wedges" and to $2.2<z<3.5$ quasars than the SDSS-III/BOSS project. Number counts and luminosity function analysis suggest that the resulting catalog is relatively complete to known quasars and is identifying new high- $z$ quasars at $z>3$. This catalog paves the way for luminosity-dependent clustering investigations of large numbers of faint, high-redshift quasars and for further machine-learning quasar selection using Spitzer and WISE data combined with other large-area optical imaging surveys.
\end{abstract}

Key words: catalogs - infrared: galaxies - methods: statistical - quasars: general

Supporting material: FITS files

\section{INTRODUCTION}

Recent years have seen considerable growth in the number and density of known actively accreting supermassive black holes in the form of active galactic nuclei (AGNs) and luminous quasars. For example, X-ray surveys now reach AGN densities of more than $9000 \mathrm{deg}^{-2}$ (e.g., Xue et al. 2011), albeit over areas of $\ll 1 \mathrm{deg}^{2}$. Spectroscopic follow-up of broadband optical imaging from the Sloan Digital Sky Survey-I/II/III (SDSS; York et al. 2000) project has expanded the number of confirmed quasars to over 270,000 objects (Schneider et al. 2010; Pâris et al. 2012) over roughly $1 / 4$ of the sky. Midinfrared (MIR) selection from Wide-field Infrared Survey Explorer (WISE) and Spitzer allows AGN selection (both unobscured and obscured) over the full sky to densities of over $60 \mathrm{deg}^{-2}$ (Stern et al. 2012; Assef et al. 2013). Deep large-area optical surveys such as the Dark Energy Survey (DES; The Dark Energy Survey Collaboration 2005) and the Large Synoptic Survey Telescope (LSST; Ivezic et al. 2008) will considerably expand the number of known AGNs even in already well-mapped areas of sky, especially at high $z$ and for low-luminosity AGNs in compact galaxies.

Our own work has sought to expand the ranks of known quasars by applying modern statistical techniques to optical imaging data instead of performing spectroscopy, increasing the number of known quasars to as many as $1,000,000$ (Richards et al. 2004, 2009a; Bovy et al. 2011) and enabling simultaneous multiwavelength (optical plus MIR) selection using those same techniques (Richards et al. 2009b). Such catalogs have enabled investigations not possible with the density of spectroscopic quasars, including cosmic magnification (Scranton et al. 2005), quasar evolution (Myers et al. 2006), the integrated Sachs-Wolfe effect (Giannantonio et al. 2012), gravitational lenses (Oguri et al. 2006), binary quasars (Hennawi et al. 2010), and dust in galaxies (Ménard et al. 2010)-particularly with rigorous mitigation of the systematics (e.g., Leistedt et al. 2013; Pullen \& Hirata 2013; Leistedt \& Peiris 2014) that are inherent to a photometric quasar sample.

The goal of this paper is to extend our previous work as follows: (1) provide both optical and MIR data that can be used to help photometrically identify even larger samples of quasars; (2) expand our pilot optical+MIR quasar selection from $\sim 24 \mathrm{deg}^{2}$ in Richards et al. (2009b) to over 10,000 deg ${ }^{2}$ by combining optical data from the SDSS and MIR data from both WISE and Spitzer-IRAC; (3) use these optical+MIR data to discover new $3.5<z<5.0$ quasars-even in areas that have already received significant attention (e.g., COSMOS and Boötes); (4) fill in the gaps of incomplete redshift from the optically targeted SDSS-I/II/III spectroscopic sample; (5) provide a discovery framework for clustering studies of high$z$ quasars within the upcoming Spitzer data within the area of SDSS Stripe 82 as part of the SpIES project (Timlin et al. 2015). 
Section 2 begins with a compilation of over 270,000 spectroscopically confirmed quasars and over 1.5 million photometrically selected quasars in the SDSS footprint. These data are the basis of our training set for further quasar discovery, and we provide this catalog in order to allow others to test their own quasar selection algorithms and to make meaningful comparison of them to ours by using the same data set. In our work, we enhance these data by matching between the SDSS optical and the MIR from WISE and Spitzer, where we have made conversions to put all of the MIR data on the same photometric system. Here we emphasize the difference between our work (which concentrates on finding new type 1 quasars, particularly at high redshift) and that of Stern et al. (2012) and Assef et al. (2013), which were designed to find both type 1 and type 2 AGNs using rigid magnitude and color cuts to minimize contamination - at the expense of highredshift quasars (Richards et al. 2009b; Assef et al. 2010).

In Section 3 we describe the construction of our optical +MIR training sets for distinguishing quasars from stars and apply our selection algorithm to a test set of objects. Our primary focus is over $3.5<z<5.0$, where MIR-only selection is most incomplete (Richards et al. 2009b; Assef et al. 2010; Messias et al. 2012); however, we also perform a selection over $2.2<z<3.5$ and $0<z<2.2$ as our method can also improve on optical-only selection, which is typically incomplete at $z \sim 2.7$ and $z \sim 3.5$ (Richards et al. 2006; Worseck \& Prochaska 2011) and reveals lower-luminosity AGNs at $z<2.2$ that optical selection alone may fail to distinguish from compact galaxies.

In Section 4 we present our catalog, including photometric redshifts. Finally, in Section 5 we make comparisons to previous work, finding that our method allows us to discover many quasars in hard-to-reach redshift ranges when using either optical-only or MIR-only selection. Our $3.5<z<5$ targets are particularly important for constraining AGN feedback models by examining the luminosity dependence of highredshift quasar clustering (Lidz et al. 2006), where current samples lack sufficient high-redshift objects over a significant range in luminosity. We have an insufficient combination of depth and areal coverage to perform this analysis with the current sample; however, such analysis can be performed with Spitzer-IRAC observations of SDSS "Stripe 82" over $\sim 110 \mathrm{deg}^{-2}$ to a depth of $\sim 6 \mu \mathrm{Jy}$ (Timlin et al. 2015). Section 5 concludes with a number counts and luminosity function analysis of the catalog and a discussion of future work.

We report photometry primarily in $\mathrm{AB}$ magnitudes, where Spitzer-IRAC Channels 1-2 are given by [3.6] and [4.5], which are the nominal wavelengths of the bandpasses in microns. For comparison with other work using Vega magnitudes, we note that the conversions between Spitzer-IRAC AB and Vega ([Vega] - [AB]) are 2.788, 3.255, 3.743, and $4.372 \mathrm{mag}$, respectively. ${ }^{9}$ For example, [3.6] $-[4.5]($ Vega $)=[3.6]-$ $[4.5](\mathrm{AB})+0.467$. For WISE, we adopt 2.699 and 3.339 as the conversions to $\mathrm{AB}$ from $W_{1}$ and $W_{2}$ Vega magnitudes, respectively, ${ }^{10}$ where the $W I S E$ central wavelengths are 3.4, 4.6, 12, and $22 \mu \mathrm{m}$ for $W_{1}, W_{2}, W_{3}$, and $W_{4}$, respectively. Cosmology-dependent parameters are determined assuming $H_{o}=70 \mathrm{~km} \mathrm{~s}^{-1} \mathrm{Mpc}^{-1}, \Omega_{m}=0.3$, and $\Omega_{\Lambda}=0.7$, in general agreement with WMAP results (e.g., Hinshaw et al. 2013).

\footnotetext{
http://irsa.ipac.caltech.edu/data/COSMOS/gator_docs/ scosmos_irac_colDescriptions.html

10 http://wise2.ipac.caltech.edu/docs/release/allsky/expsup/sec4_4h.html
}

\section{THE DATA}

To conduct our analysis, we require optical imaging data of a sample of objects that require classification; such data will constitute our test set. Some subset of those data must have already been spectroscopically classified (as quasars) and will form the basis of our quasar training set. These training and test sets will be described more fully in Section 3.1. Here we describe the origin of the data and the parameters determined from the data that are used for classification by our algorithm. Section 2.1 presents the known quasar sample used to build the training set, Section 2.2 describes the optical data, Section 2.3 discusses the infrared data, and Section 2.4 explores the redshift, magnitude, and color distributions of the matched optical-infrared data.

\subsection{Master Catalog of Quasars with SDSS Photometry}

In order to optimally select new quasars, we need the largest possible database of extant quasars with which one can build training sets. We construct such a catalog by gathering samples of spectroscopically confirmed quasars within the SDSS-I/II/ III (York et al. 2000; Eisenstein et al. 2011) footprint. Here we detail the input catalogs and the process used to combine them. We will refer to this catalog throughout the paper as the "master quasar catalog."

We started with the hand-vetted quasar catalog that concluded the SDSS-I/II project, specifically Table 5 from Schneider et al. (2010), where we have used the redshifts from Hewett \& Wild (2010) where available. The other large fraction of spectroscopic quasars comes from the the Sloan Digital Sky Survey-III: Baryon Oscillation Spectroscopic Survey (SDSSIII/BOSS) project (Dawson et al. 2013), specifically those quasars cataloged by Pâris et al. (2014) as part of "Data Release 10 ," where we used the "visual inspection" redshifts.

In addition to the standard BOSS quasars, we include a sample of 851 quasars identified on dates between late 2008 and early 2009 using Hectospec (Fabricant et al. 2005) on the MMT. The original purpose of this "MMT" quasar sample was to investigate the faint end of the quasar luminosity function (QLF) in preparation for BOSS, and quasars were targeted using deep optical data in Stripe 82 and MIR data from Spitzer where available. More details of these MMT quasars are provided in Appendix C of Ross et al. (2012a). We include all of these MMT quasars, instead of just those that were located in Stripe 82, which expands the sample compared to the 444 quasars listed in Tables 14 and 15 of Ross et al. (2012a).

Next, we add the full quasar catalog from the 2QZ project (Croom et al. 2004). ${ }^{11}$ The $2 \mathrm{dF}$ instrument provides another catalog input, namely, that from the 2SLAQ project (Croom et al. 2009), ${ }^{12}$ where we have included only objects labeled as any type of "QSO." The $2 \mathrm{dF}$ instrument has since been upgraded to the AAOmega instrument, which was used to observe objects in our third catalog from the Anglo-Australian Telescope. Specifically, we include objects from the AUS project (S. Croom et al. 2015, in preparation), including both a $K$-band limited sample and a $z>2.8$ selected sample.

We next incorporate quasar data from the AGES project (Kochanek et al. 2012), specifically using data from their Tables 5-7. We have excluded low-luminosity AGNs by

\footnotetext{
11 www.2dfquasar.org/Spec_Cat/cat/2QZ_6QZ_pubcat.txt

12 www.2slaq.info/2slaq_qso/2slaq_qso_public.cat
} 
Table 1

Master Quasar Catalog

\begin{tabular}{|c|c|c|}
\hline Column & Name & Description \\
\hline 1 & R.A. & Right ascension (J2000) \\
\hline 2 & Decl. & Declination (J2000) \\
\hline 3 & RUN & SDSS run number, see http://classic.sdss.org/dr7/glossary/index.html \\
\hline 4 & RERUN & SDSS rerun number \\
\hline 5 & CAMCOL & SDSS camera column \\
\hline 6 & FIELD & SDSS field number \\
\hline 7 & ID & SDSS ID number (within the field) \\
\hline 8 & OBJC_TYPE & SDSS object type $($ stellar $=3$, extended $=6)$ \\
\hline 9 & OBJC_FLAGS & SDSS object flags, see http://classic.sdss.org/dr7/products/catalogs/flags.html \\
\hline 10 & OBJC_FLAGS2 & SDSS object flags \\
\hline 11 & EXTINCTION & Magnitudes of Galactic extinction in ugriz \\
\hline 12 & PSFFLUX & Point-spread function flux in ugriz \\
\hline 13 & PSFFLUX_IVAR & Inverse variance of point-spread function flux in ugriz. \\
\hline 14 & PSFFLUX_CLEAN & Co-added point-spread function flux in ugriz \\
\hline 15 & PSFFLUX_CLEAN_IVAR & Inverse variance of co-added point-spread function flux in ugriz \\
\hline 16 & PSF_CLEAN_NUSE & Flag indicating whether co-added (CLEAN) flux should be used \\
\hline 17 & ZBEST & Spectroscopic and photometric redshifts from the sources indicated by SOURCEBIT \\
\hline 18 & SOURCEBIT & $\begin{array}{l}\text { Bitwise flag from } 2^{0} \text { to } 2^{13} \text { indicating the redshift source as coming from SDSS, 2QZ, AUS, AGES, } \\
\text { COSMOS, FAN, BOSS, MMT, NBCKDE, XDQSOZ, PAPOVICH, GLIKMAN, MADDOX, respectively }\end{array}$ \\
\hline 19 & SDSS_UNIFORM & Indicates whether the SDSS object fell in the "uniform" selection area; see Richards et al. (2002) \\
\hline 20 & AGES_QSO & AGES flag; see Kochanek et al. (2012) \\
\hline 21 & AGES_CODE06 & AGES flag; see Kochanek et al. (2012) \\
\hline 22 & KDE_ZPHOTLO & Minimum photometric redshift from Richards et al. (2009a) \\
\hline 23 & KDE_ZPHOTHI & Maximum photometric redshift from Richards et al. (2009a) \\
\hline 24 & KDE_ZPHOTPROB & Photometric redshift probability from Richards et al. (2009a) \\
\hline 25 & KDE_LOWZORUVX & Flag indicating a UV-excess or low-redshift source; Richards et al. (2009a) \\
\hline 26 & XDQSOZ_PEAKPROB & Peak of the redshift probability from Bovy et al. (2012) \\
\hline 27 & XDQSOZ_PEAKFWHM & FWHM of the redshift peak from Bovy et al. (2012) \\
\hline 28 & XDQSOZ_NPEAKS & Number of peaks in the Bovy et al. (2012) photo- $z$ distribution \\
\hline
\end{tabular}

(This table is available in its entirety in FITS format.)

requiring qso $=1$ from Table 5. Quasars from another deep, wide area, namely, COSMOS (Scoville et al. 2007b), ${ }^{13}$ have also been included in our sample, where the data were limited to type 1 objects (Lilly et al. 2007; Trump et al. 2009).

To increase the number of rare, very high redshift quasars, we also include $65 z>5.8$ quasars from Fan et al. (2006) and Jiang et al. (2008). The master quasar catalog was built before a large number of $z \sim 5$ quasars were cataloged in Stripe 82 by McGreer et al. (2013), but we recover 49 of the 65 that are bright enough to have matching MIR photometry.

Our master quasar catalog is rounded out by a few smaller samples of objects meant to extend the range of properties covered. This includes the "BROADLINE" objects from Table 5 of Papovich et al. (2006), the $z \sim 4$ quasars from Table 5 of Glikman et al. (2010), and $K X$-selected quasars at $z>1$ from Maddox et al. (2012, Tables 4 and 6).

There may yet be some known type 1 quasars within the SDSS footprint that we have not included in our master quasar catalog; however, they should mostly be small samples of objects that are already represented or much brighter than the SDSS flux limits (e.g., 3C 273 and most "PG" quasars from Schmidt \& Green 1983).

All of the above objects are spectroscopically confirmed quasars; however, many more likely quasars have been identified photometrically. As that information also has value in considering identification of new quasars, we have included objects listed in the photometric quasar catalogs of both

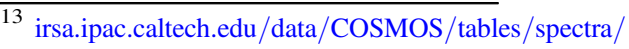

Richards et al. (2009a, NBCKDE) and Bovy et al. (2011, XDQSO).

These individual tables are merged together, and a flag is set to indicate the origin. The flag values run from 0 to 13 as follows, where spectroscopic redshifts from earlier catalogs in the list trump later catalogs when there is a duplication: SDSS, 2QZ, 2SLAQ, AUS, AGES, COSMOS, FAN, BOSS, MMT, NBCKDE, XDQSOZ, PAPOVICH, GLIKMAN, MADDOX.

For the benefit of those wishing to make use of this master catalog, we make it available in Table 1. The columns are as follows: (1) R.A. (degrees); (2) decl. (degrees); (3-7) SDSS run, rerun, camcol, field, and id; ${ }^{14}$ (8) the SDSS morphology (OBJC_TYPE); (9-10) code indicating SDSS data quality (OBJC_FLAGS and OBJC_FLAGS2); (11) SDSS Galactic EXTINCTION in all five bands; (12) the SDSS flux as measured from point-spread function (PSF) fitting (in nanomaggies) in all five bands; (13) the inverse variance of the PSF flux in all five bands; (14) the co-added SDSS PSF flux for those objects observed in multiple epochs, (15) the inverse variance for column 14; (16) PSF_CLEAN_NUSE, which is an indication of whether there are multiple epochs of imaging data (values larger than 1 indicate that we have used the "CLEAN" [i.e., co-added] values of the PSF flux in our analysis); (17) ZBEST, which indicates the redshift determined from each of the sources of data described in (18) SOURCEBIT (numbered 0-13 in the order given above); (19) SDSS_UNIFORM, which

\footnotetext{
$\overline{14}$ These and other SDSS-related information are described in more detail at https://www.sdss3.org/dr9/imaging/imaging_basics.php.
} 
indicates whether the SDSS object fell in the "uniform" selection area as described by Richards et al. (2002); (20-21) codes from the AGES survey that we used to reject low-redshift AGNs from our training set; (22-25) photometric redshift information from the NBCKDE photometric quasar catalog (Richards et al. 2009a); (26-28) photometric redshift information from the XDQSO photometric quasar catalog (Bovy et al. 2012).

\subsection{Optical Data}

Over more than $10 \mathrm{yr}$, the SDSS used a sophisticated telescope (Gunn et al. 2006) fitted with a large-field-of-view camera (Gunn et al. 1998) to take exposures through ugriz filters (Fukugita et al. 1996). For the training and testing sets in this paper, we use the "Data Release 9" (DR9) versions of this SDSS imaging (Ahn et al. 2012). DR9 included the latest astrometric and photometric calibrations for imaging in the original northern SDSS footprint and in the southern footprint that was added as part of SDSS DR8 (Aihara et al. 2011). Specifically, we use the versions of the SDSS imaging provided in the calibobj or "data sweep" files ${ }^{15}$ that are discussed in Blanton et al. (2005). We limit the data sweeps to only objects that are PRIMARY in SDSS imaging (e.g., see Table 5 of Stoughton et al. 2002), but do not further restrict our optical sources using cuts on image quality flags at this stage (any additional flag cuts are described in the relevant sections of this paper). We use such PRIMARY sources from the SDSS data sweep files as our test data and also match our heterogeneous master training catalog of quasars (described in the previous section) to PRIMARY objects from these data sweeps.

While the spectroscopic identifications that we tabulate have a heterogeneous origin, one advantage of the catalog of quasars that we have built is that their optical imaging is derived solely from the SDSS imaging camera (Gunn et al. 1998), providing a homogeneous aspect to the data set.

All of the optical magnitudes reported in the catalog are asinh PSF magnitudes (Lupton et al. 1999) corrected for dust extinction using the coefficients given by Schlafly \& Finkbeiner (2011). Fluxes are reported in nanomaggies without any dust extinction correction. The full list of cataloged parameters is given in Table 1 for the master quasar catalog and Section 4 for our quasar candidate catalog; further information on each source is publicly available.

\subsection{Infrared Data}

To create our MIR data set, we begin by merging large areas of relatively deep Spitzer-IRAC data (Fazio et al. 2004) with shallower but wider-area WISE data (Wright et al. 2010). This has the advantage of allowing us to probe both a wide area and relatively deep (in a fraction of that area).

The WISE data come from the AllWISE data release, ${ }^{16}$ where we have kept only objects with both $W_{1}$ and $W_{2}$ detections and have excluded objects that do not meet the following quality control criteria: $\mathrm{w} 1 \mathrm{flg}<=1 \&$ \& $\mathrm{w} 2 \mathrm{flg}<=1$ (to avoid sources with bad pixels or that are upper limits), CC_flags $===^{\prime} 0000^{\prime}$ (to avoid objects affected by diffraction spikes, ghosts, latent images, and scattered light), ext_flg $==0$ (to limit to MIR point sources), and w1snr $>2 \& \& \mathrm{w} 2 \mathrm{snr}$

\footnotetext{
15 http://data.sdss3.org/datamodel/files/PHOTO_SWEEP/RERUN/ calibObj.html

16 http://wise2.ipac.caltech.edu/docs/release/allwise/
}

$>2$ (to limit to objects that are well detected in both $W_{1}$ and $\left.W_{2}\right) .{ }^{17}$ By matching known SDSS quasars to AllWISE, we estimate that these cuts cull $9.6 \%, 3.0 \%, 0.6 \%$, and $0.2 \%$ of real sources, respectively. This incompleteness is corrected in our number counts and luminosity function analysis in Section 5.

The Spitzer catalogs include (1) the SWIRE data (Lonsdale et al. 2003), (2) the XFLS data (Lacy et al. 2005), (3) the COSMOS data (Sanders et al. 2007), (4) our own pilot sample of Spitzer-IRAC data centered on known high- $z$ quasars in SDSS Stripe 82 (data tabulated in Krawczyk et al. 2013), (5) the SDWFS data in the Boötes field (Eisenhardt et al. 2004), and (6) the SERVS data (Mauduit et al. 2012). The SWIRE, XFLS, COSMOS, and Spitzer Deep, Wide-field Survey (SDWFS) data are the same data used in Richards et al. (2009b); see that paper for more details. Boötes data are taken from Ashby et al. (2009), specifically SDWFS_ch1_stack. v34.txt, adopting the aperture-corrected $4^{\prime \prime}$ (diameter) flux densities. This catalog corresponds to a depth of $12 \times 30 \mathrm{~s}$, and we have limited to objects detected in both Channels 1 and 2 and with SExtractor flags of 0 or 2 . Vega magnitudes have been converted to $\mu \mathrm{Jy}$. The SERVS data are described in detail in Mauduit et al. (2012).

Our Stripe 82 data include pointed observations of over 300 known $z>2$ quasars in the SDSS Stripe 82 field (Annis et al. 2014; Jiang et al. 2014) and were processed in a manner similar to that which was used for the SWIRE data set. Photometry for these sources is tabulated in Krawczyk et al. (2013). We report fluxes in a 1"!9 aperture radius.

For all of the above data sets, we have included all objects that are not flagged by SExtractor (Bertin \& Arnouts 1996) as blended in either IRAC Channel 1 or Channel 2, and we have applied no explicit flux limits to the individual catalogs. Flux densities have been converted to $\mu \mathrm{Jy}$ if the original data have other units. We report errors that have been increased by $3 \%$ (10\% for XFLS) in quadrature since SExtractor only reports the rms at the image position; this is consistent with Donley et al. (2012, Section 4).

We would like to be able to use MIR measurements from both WISE and Spitzer; however, photometry from these two spacecraft are on different photometric systems. There is strong similarity in the two shortest wavelength filters of the systems, but a correction needs to be applied. As such, the WISE data have been converted from Vega magnitudes on the WISE system to $\mu \mathrm{Jy}$ in the Spitzer-IRAC system using color terms appropriate for each of the individual objects (based on their $W_{1}-W_{2}$ colors). This process is important for allowing us to treat the WISE and Spitzer data equivalently. As the $W_{3}$ and $W_{4}$ data are much shallower than $W_{1}$ and $W_{2}$, we have only tabulated the $W_{1}$ and $W_{2}$ photometry and have only kept objects with detections in both of those bands.

As an illustration of our conversion of the WISE Vega system to Spitzer AB, we convert the $W_{1}-W_{2}$ (Vega) $=0.8$ color cut used by Stern et al. (2012) to the Spitzer AB system. First, we find that

$$
\begin{aligned}
W_{1}(\text { Vega })-W_{2}(\text { Vega })= & \left(W_{1}(\mathrm{AB})-2.699\right) \\
& -\left(W_{2}(\mathrm{AB})-3.339\right)
\end{aligned}
$$

\footnotetext{
17 See http://wise2.ipac.caltech.edu/docs/release/allwise/expsup/sec2_1a. $\mathrm{html}$ for a detailed explanation of these parameters.
} 
so that the above cut is $W_{1}-W_{2}(\mathrm{AB})=W_{1}-W_{2}$ (Vega) $0.64=0.16$. We have then created a look-up table for the conversion of WISE AB magnitudes to Spitzer AB magnitudes as a function of color (assuming a power-law spectral energy distribution [SED]). In general, these corrections are small for $W_{1}$ and $W_{2}$ (see Wright et al. 2010, Table 1). We find that at $W_{1}-W_{2}(\mathrm{AB})=0.16,[3.6]=W_{1}(\mathrm{AB})-0.028$ and $[4.5]=$ $W_{2}(\mathrm{AB})+0.013$, so that $W_{1}-W_{2}(\mathrm{Vega})=0.8$ is equivalent to $[3.6]-[4.5](\mathrm{AB})=0.119$. Similarly, we can convert a $W_{2}$ (Vega) $=15.05$ magnitude cut (at this color) to Spitzer $\mathrm{AB}$ as follows: [4.5] $(\mathrm{AB})=W_{2}(\mathrm{Vega})+3.339+0.013=$ 18.402. We illustrate these cuts in Section 3.1, where for the sake of simplicity we have ignored the color dependence of the magnitude limit. As the agreement with Spitzer photometry has significantly improved for the AllWISE data release as compared to the older, All-Sky WISE data, we have not further corrected for the remaining offsets. The typical AllWISE limits are $54 \mu \mathrm{Jy}$ in $W_{1}$ or 16.9 in Vega mag and $71 \mu \mathrm{Jy}$ in $W_{2}$ or 15.9 in Vega mag, but they depend on location due to WISE's polar orbit. In AB mag, these limits are 19.6 and 19.3. See the AllWISE Explanatory Supplement ${ }^{18}$ for a discussion of how the Spitzer and WISE differences, [3.6] $-W_{1}$ and [4.5] $-W_{2}$, behave as a function of magnitude and for information on how the WISE sensitivity changes with coordinate.

We generate a single merged MIR catalog by matching the above data sets using a $2^{\prime \prime}$ matching radius, with priority being given to objects from the individual catalogs as follows: SERVS, SWIRE, COSMOS, SDWFS, XFLS, Stripe 82, and WISE. That is, an SERVS detection will overwrite an SWIRE detection. Only one Spitzer detection of each object was allowed, and a flag was set to indicate which catalog the photometry comes from. However, if there are data from both WISE and Spitzer, we have also kept the WISE data for reference.

This final MIR catalog is then matched to the SDSS-III imaging data using a $2^{\prime \prime}$ matching radius. No explicit flux limits have been applied. Dust extinction has been corrected as $A_{[3.6]}=0.197 E(B-V)$ and $A_{[4.5]}=0.180 E(B-V)$, consistent with Cardelli et al. (1989) as reported by the NASA/IPAC Infrared Science Archive. ${ }^{19}$

The full SDSS-III footprint lacks deep near-IR imaging, since the Two Micron All Sky Survey (2MASS; Skrutskie et al. 1997) is too faint to provide counterparts for the bulk of our quasar sample. However, when available, near-IR data are very useful for improving photometric redshift (photo-z) estimates. Thus, while we do not use near-IR data for our quasar selection algorithm, we do match our optical catalog to near-IR catalogs from the regions of sky covered by the UKIRT Infrared Deep Sky Survey (UKIDSS; Lawrence et al. 2007) and the Vista Hemisphere Survey (VHS; McMahon 2012). We used a matching radius of $1^{\prime \prime}$ and included only objects that have measurements in each of $J, H$, and $K$. While these near-IR data are not simultaneous with the optical or MIR data, which causes some scatter in the color distributions, even simultaneous observed-frame multiwavelength (and thus multidistance scale) data would not be simultaneous in the rest frame.

\footnotetext{
18 http://wise2.ipac.caltech.edu/docs/release/allwise/expsup/sec2_3a.html

19 irsa.ipac.caltech.edu
}

Figure 1 shows the relative limits of the MIR and near-IR data as compared to the optical for a typical quasar SED (Krawczyk et al. 2013). High- $z$ quasars found from SDSS photometry with $i<20$ are expected to be detected in AllWISE. They should also be detected by UKIDSS and may be detected by GALEX in the bluest bandpass. Quasars closer to the SDSS photometric limit (for single-epoch data) can be much fainter than the AllWISE, UKIDSS, and VHS limits, which will limit the completeness of this catalog. Fainter quasar candidates are limited by the depth of AllWISE (or the area of Spitzer).

\subsection{Diagnostics}

Here we provide some diagnostic plots to illustrate the range of optical and MIR properties spanned by our choice of data. Figure 2 shows the redshift distribution for all of the objects in our master quasar catalog, including those objects where only optical photometry is available and those objects where MIR photometry exists. The peaks in redshift in this figure represent selection effects. The SDSS-DR7 quasar sample peaked at $z \sim 1.5$, while the SDSS-DR10 quasar selection was optimized for $z \sim 2.5$, with contamination coming at $z \sim 0.8$. Most of the losses of IR-matched objects at low redshift are due to the flag cuts imposed on the WISE data. At high redshift, the difference between the focus of our work (not relying on MIR color cuts) and that of Assef et al. (2013; which utilizes MIR color cuts) is readily apparent.

Figure 3 shows the magnitude distribution of the objects in the master catalog. The peaks in the distribution are caused by a combination of magnitude limits: the SDSS-DR7 quasar sample had a $z<3$ magnitude limit of $i<19.1$ and a $z>3$ limit of $i<20.2$, while SDSS-DR10 probed to $g<21.85(i \sim 22)$. Although adding MIR photometry is very powerful for AGN selection, it is also responsible for reducing the completeness to known quasars by a factor of $\sim 2$ by $i=20$. Up to $i \sim 19$, over $80 \%$ of our quasar sample includes IR measurements from WISE or Spitzer. Most of the losses at bright magnitudes occur as a result of our attempts to restrict ourselves to the highest-quality WISE data as noted above. The fraction of bright quasars with IR matches is roughly consistent with the expected loss of $\sim 13 \%$ of sources due to the flag cuts on the WISE data and the fraction found by $\mathrm{Wu}$ et al. (2012). That is, the curves in the insets of Figures 2 and 3 should be shifted up by 0.13 to correct for objects removed owing to flag cuts. The dotted lines show the effect of the Assef et al. (2013) reliability cuts relative to the objects in our training set (dashed lines).

Figure 4 shows the quasar colors as a function of redshift. In addition to the data points, we also depict the mean colors as a function of redshift for both the full optical sample and the more limited optical+MIR sample. Overall, there is good agreement between the samples.

\section{CLASSIFICATION}

In Section 2 we tabulated quasars both with and without MIR photometry; for the remainder of this paper we will consider only the optical+MIR data set. After building training and test sets (Section 3.1) in a similar manner to that described in Richards et al. (2009b), we will apply the same Bayesian selection algorithm (Section 3.2) described in our previous papers, and then we will describe the selection results (Section 3.3). 


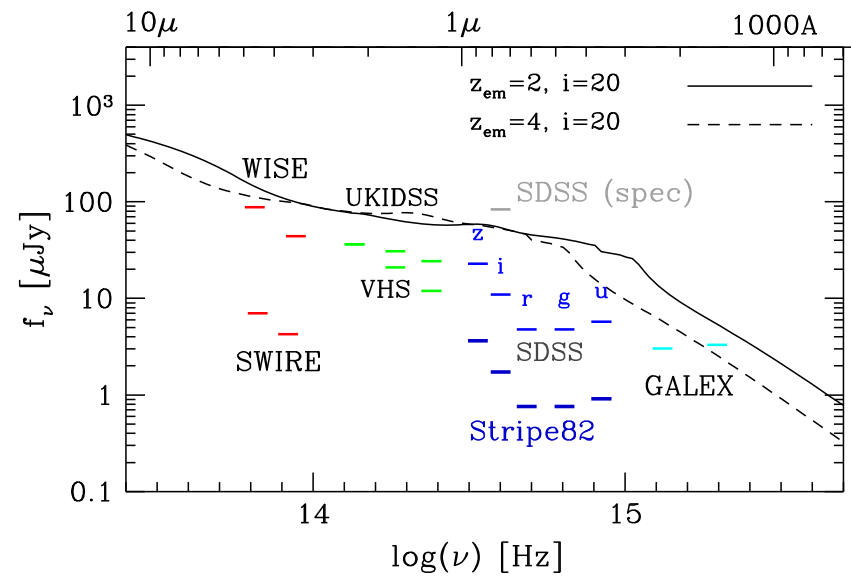

Figure 1. Relative limits of the multiwavelength data. The bars indicate the effective wavelength of the bandpasses, but are not scaled to represent the size of the bandpass. Red indicates MIR data from AllWISE and Spitzer-SWIRE, green indicates the limits of UKIDSS and VHS, blue shows the depth of both single-epoch and multi-epoch (Stripe 82) SDSS photometry, while cyan gives the limits of the GALEX AIS survey. Two example quasar SEDs (from Krawczyk et al. 2013) are given for $z=2$ (solid black line) and $z=4$ (dashed black line), both corrected for Lyman series extinction and normalized to $i=20$, which is roughly the limit of SDSS spectroscopy for high redshift (it is $i=19.1$ for low redshift, which is shown in gray).

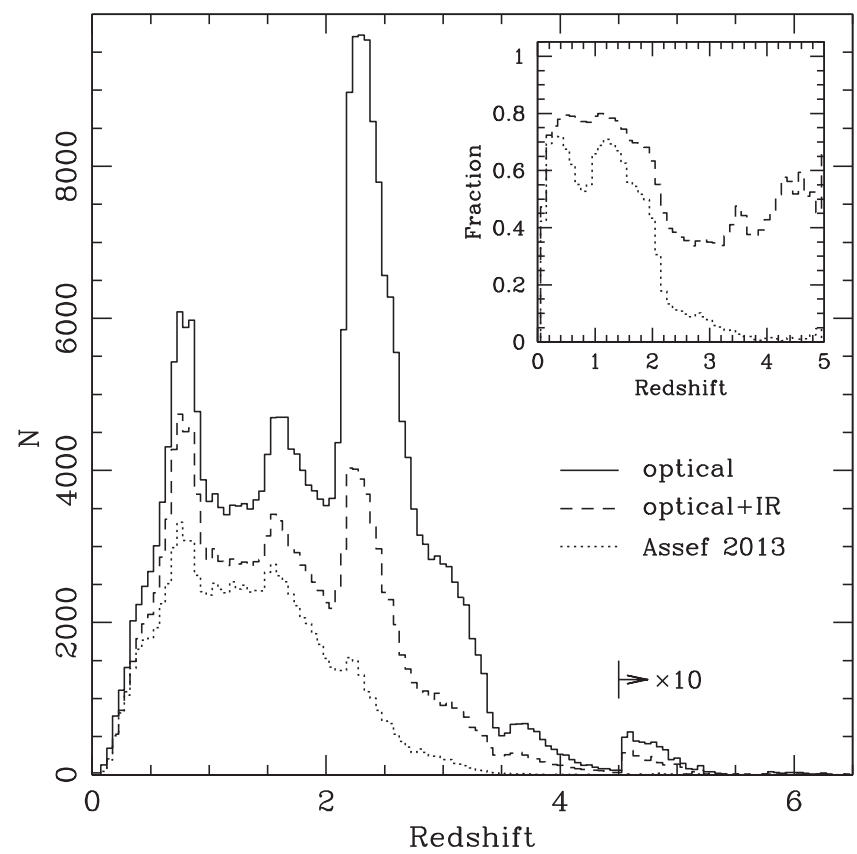

Figure 2. Redshift distribution of the full spectroscopic quasar sample (solid line; 274,329 quasars), the IR-matched sample (dashed line; 157,701 quasarsthe parent sample of our quasar training sets), and the IR-matched sample with the 75\% reliability limit from Assef et al. (2013) imposed (dotted line). Beyond redshift 4.5 the distributions have been scaled by a factor of 10 to better show the high- $z$ part of the samples. The inset gives the ratio of the dashed line to the solid line and the dotted line to the solid line. Losses at low redshift are dominated by flag cuts $(\sim 13 \%$, independent of redshift). Further losses at high redshift are primarily due to implicit (dashed line) or explicit (dotted line) magnitude limits of the subsamples, as can be seen in Figure 3.

\subsection{Training and Test Sets}

Starting with the matched optical+MIR photometry (for both known quasars and all SDSS-DR10 sources), we create the test set (objects to be classified) along with the quasar and nonquasar ("star") training sets as follows.

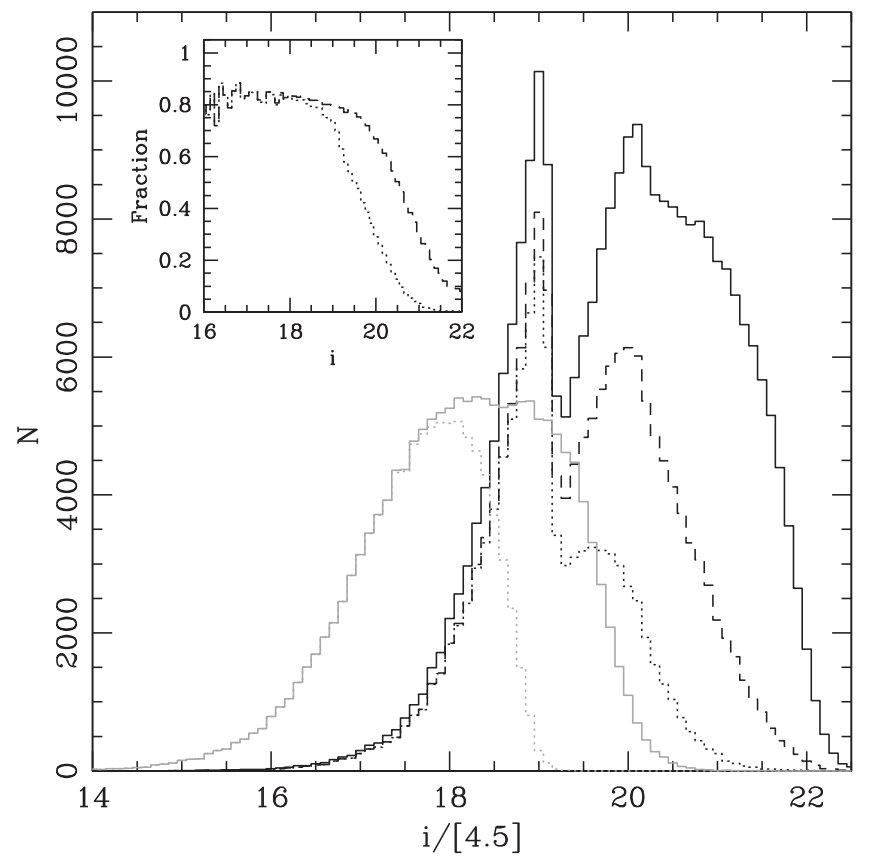

Figure 3. The $i$-band magnitude distribution of the full spectroscopic quasar sample (solid black line), the IR-matched sample (dashed black line; the parent sample of our quasar training sets), and the IR-matched sample with the $75 \%$ reliability limit from Assef et al. (2013) imposed (dotted black line). The inset shows the ratio of the latter two samples to the full sample, demonstrating that our matching to WISE (and/or Spitzer) photometry is over $80 \%$ complete to $i \sim 19$ (dashed line) and that our greater sensitivity to high-redshift quasars relative to Assef et al. (2013; dotted line) is largely due to probing deeper. The gray histograms in the main panel show the distribution in [4.5] for our full training set (solid) and after imposing the 75\% reliability cut of Assef et al. (2013; dotted).

We first restrict the data to objects that are expected to have "clean" photometry, which, for our purposes, we define based on whether or not they have any of the following SDSS imaging quality flags set: INTERP_PROBLEMS, DEBLEND_PROBLEMS, NOT_BINNED1, EDGE, BRIGHT, SATUR, MOVED, BLENDED, NODEBLEND, and NOPROFILE. These flags are fully defined in Table 9 of Stoughton et al. (2002) except for INTERP_PROBLEMS, DEBLEND_PROBLEMS, and MOVED, which are detailed in Richards et al. (2002) and/or are further discussed in Appendix A of Ross et al. (2012a). Objects must also have flux values of $<1000$ nanomaggies $\left(m_{\mathrm{AB}}>15\right)$ in all bands to be included, since brighter fluxes can lead to saturated pixels. However, we have made this cut before applying any dust extinction corrections, so objects that are intrinsically brighter than $m_{\mathrm{AB}}=15$ but that are not saturated in the images are kept.

If good co-added (multiepoch) photometry is reported in all bands (as indicated by PSF_CLEAN_NUSE $>0$ ), ${ }^{20}$ then we retain the co-added fluxes (and errors); otherwise, the singleepoch fluxes are used. To handle the problem of negative fluxes, we have used the asinh magnitude prescription of Lupton et al. (1999).

Initially, our classification included both point and extended (optical) sources, as have our previous catalogs. Later we will restrict our analysis to just the point sources. At this point, the test set consists of all the photometry from all of the "good"

\footnotetext{
${ }^{20}$ Again see http://data.sdss3.org/datamodel/files/PHOTO_SWEEP/ RERUN/calibObj.html for descriptions of the format of the data sweeps files that we use.
} 


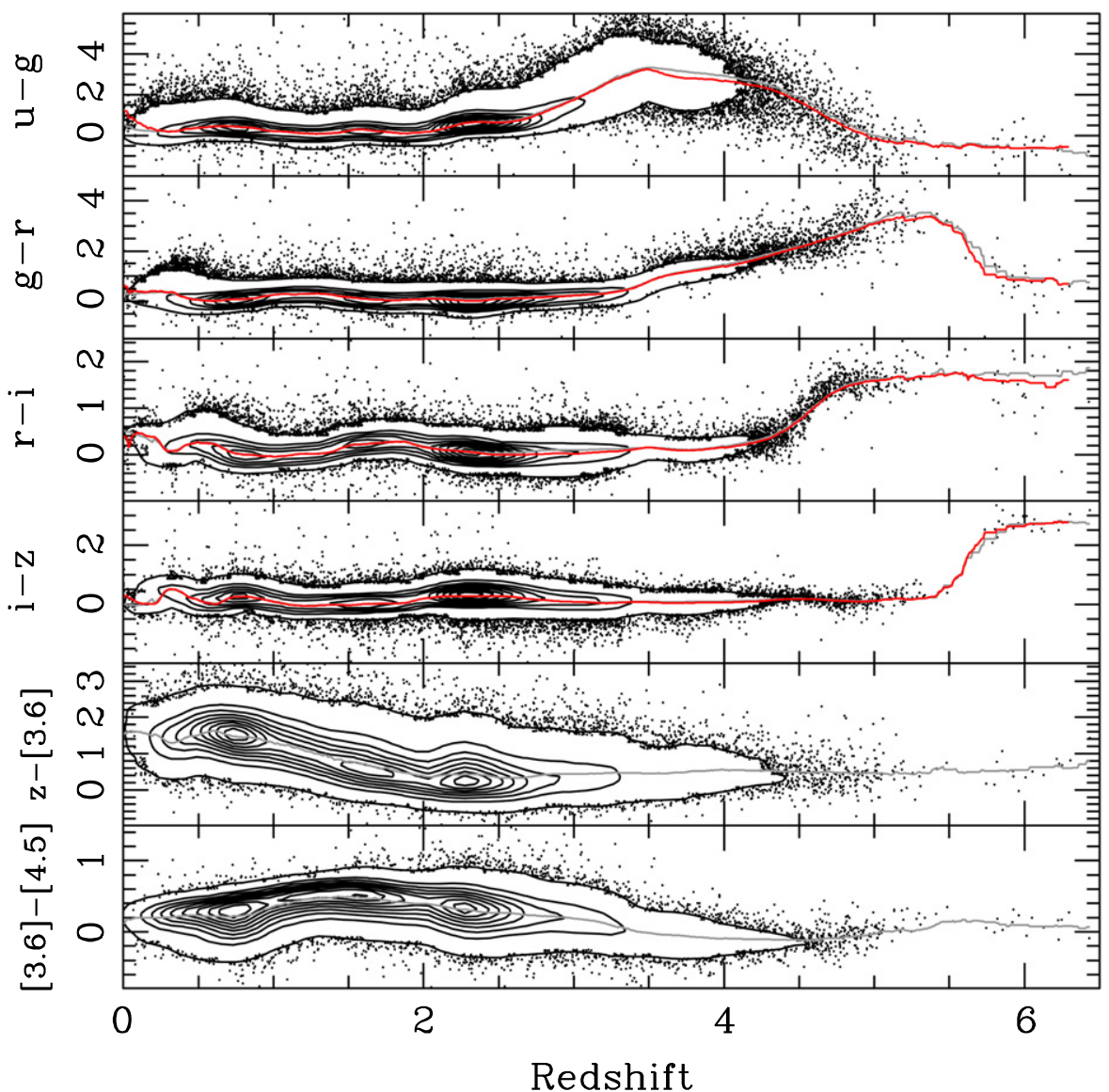

Figure 4. Color vs. redshift for the spectroscopic quasar sample. Black (linear) contours and dots show the color distributions of the individual objects. The top four panels include all of the spectroscopic objects; the bottom two panels contain only those matching to the IR sample. Lines give the mean color-redshift relations (which are used to compute the photometric redshifts). The red line is for all of the optical data, while the gray line shows the mean for the objects that additionally have IR matches. In the top four panels there is good agreement between the red and gray lines (and thus between the quasars with and without matching IR photometry).

point and extended sources described above. No further restrictions are placed on the objects that we attempt to classify. The classification parameters are the set of adjacent colors determined from each of the five optical and two mid-IR magnitudes that we catalog, specifically $u-g, g-r, r-i$, $i-z, z-$ [3.6], and [3.6] - [4.5]. In all there were 50,225,630 objects in the test set.

The quasar training set is the subset of the test set for which there is a match in the master quasar catalog with a spectroscopic redshift (i.e., we have not included photometric quasars) as noted in Section 2.1. The "stars" training set is again a subset of the test set. Here sources matched to known (spectroscopic) quasars are excluded. The final stars training set is a randomly selected sample of $\sim 700,000$ objects (taking those objects where the hundredths and thousandths digits of the IRAC $\mathrm{CH} 2$ flux density were "01"). The vast majority of these objects lack spectroscopic classification as stars; thus, these are not only stars, but can be (compact) galaxies (and previously unidentified quasars); see the discussion of the cleaning process below. Thus, "stars" in this context is shorthand meaning optical point sources that have not been classified as quasars in the redshift range we are trying to select.

In practice, we have actually made three pairs of quasar and star training sets, as quasar colors change considerably at high redshift, and it is best to treat them as separate populations.
Thus, the quasar training sets are created by parsing through the quasars and keeping only those within the redshift range of interest. Quasars outside of that redshift range are put into the "stars" training set. The three ranges used are $0<z<2.25$ (11,984 quasars), $2.15<z<3.55$ (45,561 quasars), and $3.45<z<5.5$ (3321 quasars), where the overlap is to minimize the loss of objects near the redshift boundaries and we stop at $z=5.5$ since selecting higher redshifts generally requires additional care (Fan et al. 2006). We will refer to objects selected from the use of training sets focusing on these redshift ranges as "low-z," "mid- $z$," and "high- $z$ " throughout the rest of the paper.

Figure 5 presents the optical colors (and a magnitude) of the objects in our training sets. For the star training set, we show only the low- $z$ training set, which includes quasars above $z=2.2$. All three quasar training sets are shown. Similarly, Figure 6 gives the MIR colors of the training set objects. Here we include the color-magnitude cuts (solid black line) used by Stern et al. (2012) to select their quasar sample in addition to the (somewhat more inclusive) $75 \%$ reliability selection (solid yellow curve) of Assef et al. (2013). Comparison of these curves to the distribution of high-redshift quasars illustrates their bias against such objects as shown in Section 2.4. This reflects a conscious choice to be sensitive to both type 1 and type 2 AGNs without significant contamination from inactive galaxies. Our approach is complementary in that we will 

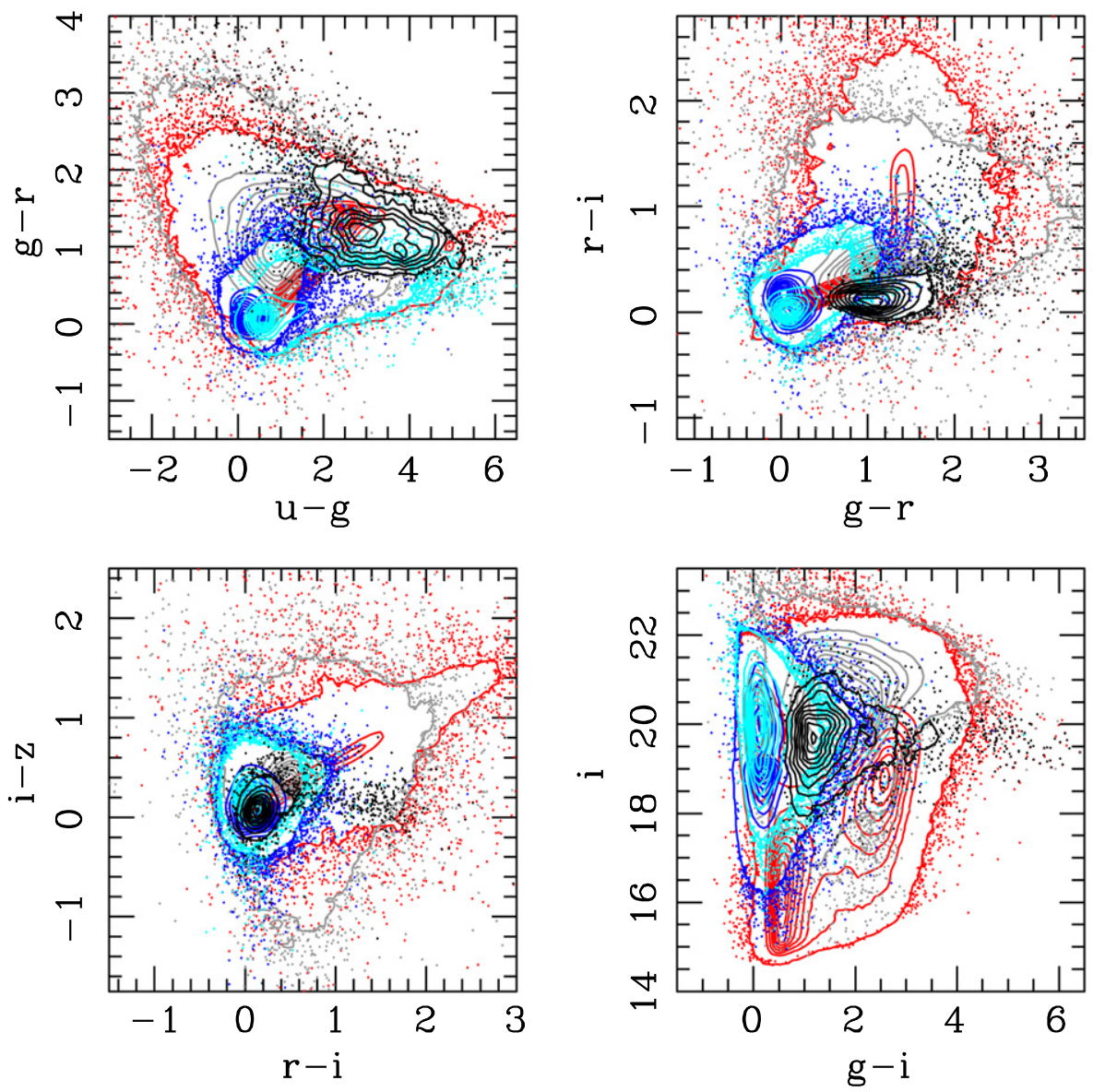

Figure 5. Optical colors of training set objects. Point sources are in red, extended sources in gray, high- $z$ quasars in black, mid- $z$ quasars in cyan, and low- $z$ quasars in blue. Extended sources are not explicitly included in the training set but are shown here for reference given that separation of point and extended sources is not perfect (particularly at faint magnitudes).

endeavor to be as complete as possible to high-redshift type 1 quasars, at the expense of type 2 quasars. The green lines in Figure 6 depict the cuts that we will use to reduce stellar contamination from the test sets as shown in Section 3.3. We duplicate them here to emphasize that they would throw out relatively few of the training set objects.

\subsection{Application of the Algorithm}

As described in more detail in Richards et al. (2004, 2009a, 2009b), our algorithm requires that we compute a "bandwidth" that best describes the range of colors of each object class. This is akin to determining the best bin size to represent one's data in a histogram: having too many bins leaves too few objects in each bin, while having too few bins oversmooths the data and causes a loss of information. Thus, the bandwidth is essentially a smoothing parameter for the color distributions. These bandwidths are determined by a selfclassification step, choosing the bandwidth that yields the most complete recovery of known quasars with the smallest contamination from stars. As in our previous work, we first perform an initial self-classification of the training sets, and then we throw out objects initially classified as quasars from the star training set (since we expect our star sample to be contaminated by those very objects that we wish to recover where other algorithms have failed). The final bandwidth is determined from the original quasar training set and the "cleaned" star training set. An example "heat map" showing the minimization of the bandwidths for self-classification of stars and quasars in the high- $z$ training sets is shown in Figure 7. Optimal bandwidths were computed for each of the low $-z$, mid- $z$, and high- $z$ training sets.

The only other free parameter in our classification is the Bayesian stellar prior, which represents our expectation of what fraction of objects are really stars. For low- $z$ classification this was set to $98 \%$ (that is, we expect $98 \%$ of the objects in the test set to be "stars"). For the mid- $z$ classification it was set to $99.9 \%$, reflecting the lower density of quasars in this redshift range as compared to lower redshift. Finally, for high-z classification, it was set to $99.99 \%$. These numbers are estimated from the ratio of the number of objects in the test set to the number of objects in the training set, which provides a conservative estimate of the quasar fraction. These star priors demonstrate the level to which quasar classification is a "needle in a haystack" problem that requires methods more sophisticated than simple color cuts. Note that small changes in the prior only make small changes in the number of quasars selected. For example, in the low- $z$ case, lowering the stellar prior by $1 \%$ does not increase the number of quasar candidates by $1 \%$ of the test set (roughly a half million objects); rather, we find that it changes the number by roughly $1 \%$ of the quasar candidates ( 7000 objects). 

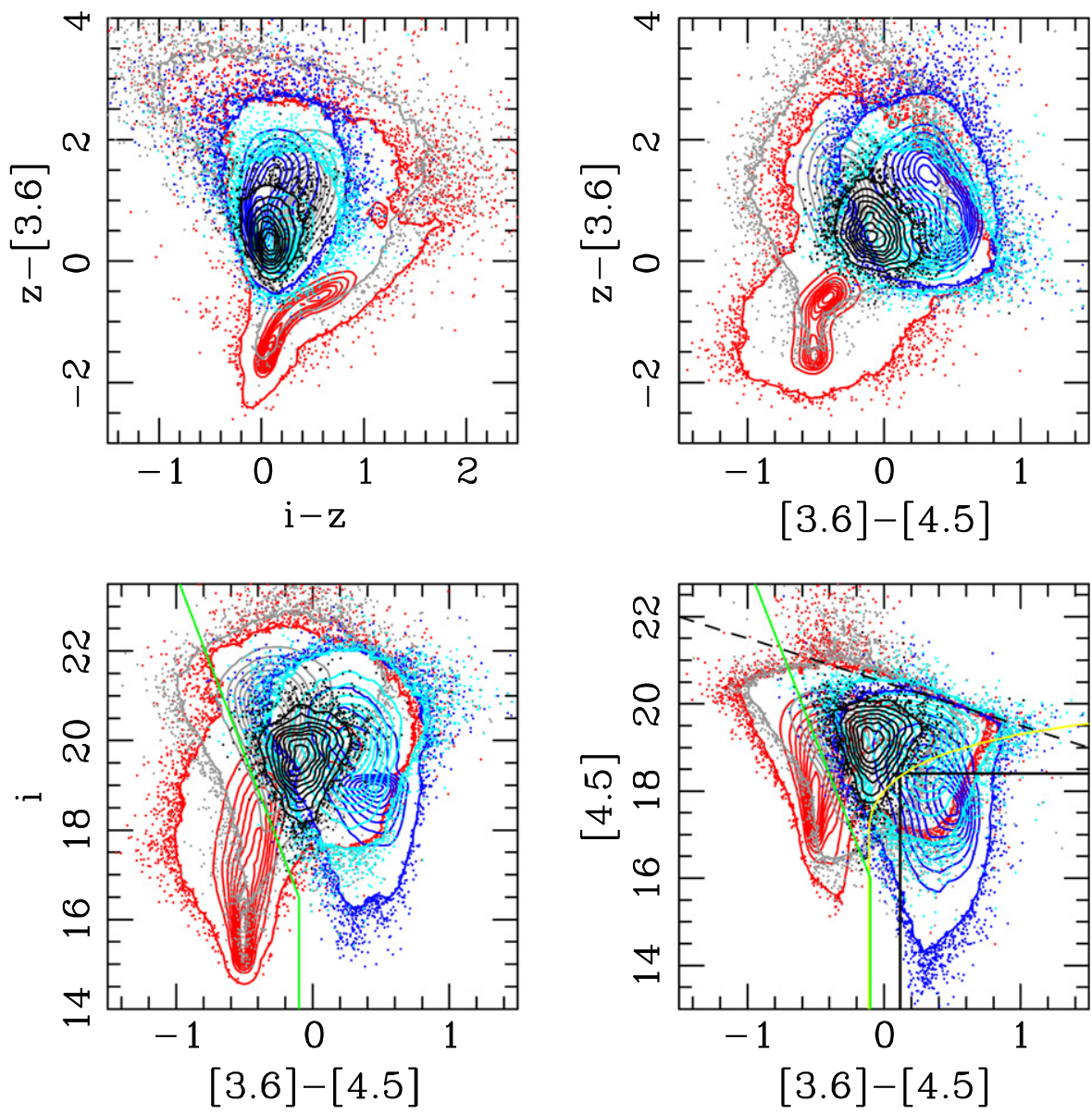

Figure 6. MIR colors of training set objects. Point sources are in red, extended sources in gray, high- $z$ quasars in black, mid- $z$ quasars in cyan, and low- $z$ quasars in blue. The dashed black line shows the detection limit as a function of color for a theoretical object with [3.6] $=20.5$. The solid black lines indicate the color and magnitude limits of the Stern et al. (2012) selection in AB magnitude space, while the yellow curve gives the 75\% reliability selection from Assef et al. (2013). The green lines in the bottom panels give our own cuts that are intended to reduce stellar contamination; these are not applied to the training sets but are shown here for comparison to the test set output.

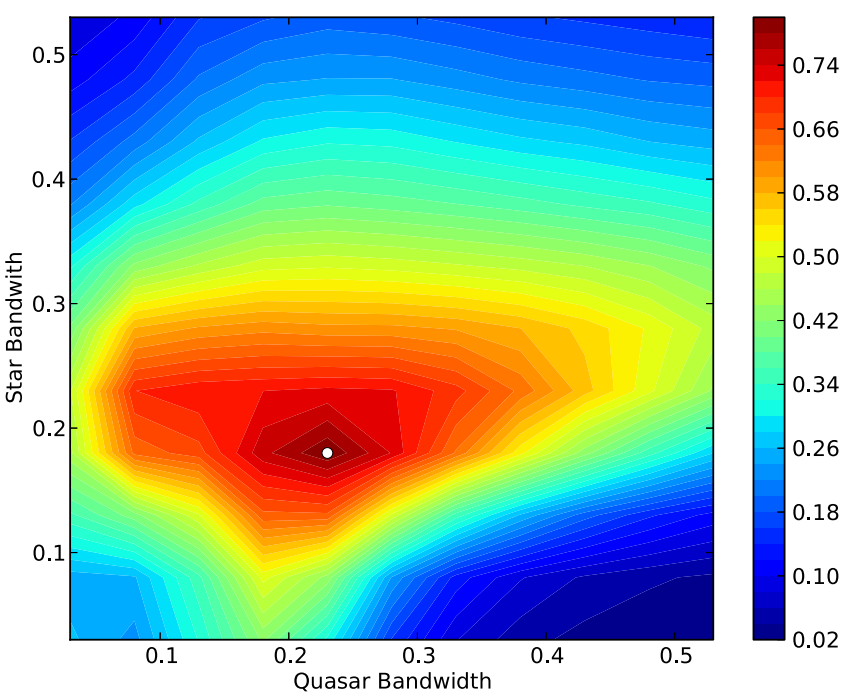

Figure 7. Graphical depiction of the search for optimal bandwidths for the star and quasar training sets. The color bar indicates the "rating" of each bandwidth pair, which is determined by the product of the self-completeness and efficiency. The optimal bandwidth in this case (the high- $z$ training set) was found to be $(0.23,0.18)$ for (quasars, stars).

\subsection{Classification Results}

Here we present the results of our classification. This process is an extension of the $8 \mathrm{D}$ (optical plus MIR colors) selection described in Richards et al. (2009b), using the algorithm described in more detail by Richards et al. (2004, 2009a).

Our algorithm can roughly be summarized as choosing objects for which

$$
P(\text { colors } \mid \text { quasar }) P(\text { quasar })>P(\text { colors } \mid \text { star }) P(\text { star }),
$$

where $P($ star $)$ is the stellar prior, $P$ (quasar) is $1-P($ star), and $P$ (colors I quasar) is the probability of an object having certain colors given that it is known to be a quasar (and similarly for the stars). In practice, we have performed this classification in a discrete binary fashion using $k d$ trees; see Gray et al. (2005) and Riegel et al. (2008). However, we compute the continuous probabilities for all of the objects that satisfy the initial binary selection criterion and we report those values in the final catalog as they can sometimes be useful in post assessment of the classification accuracy.

This process identified $1,317,677$ objects as low- $z$ quasar candidates, 804,342 as mid- $z$ quasar candidates, and 48,324 objects as high- $z$ quasar candidates. These amount to $2.6 \%$, 

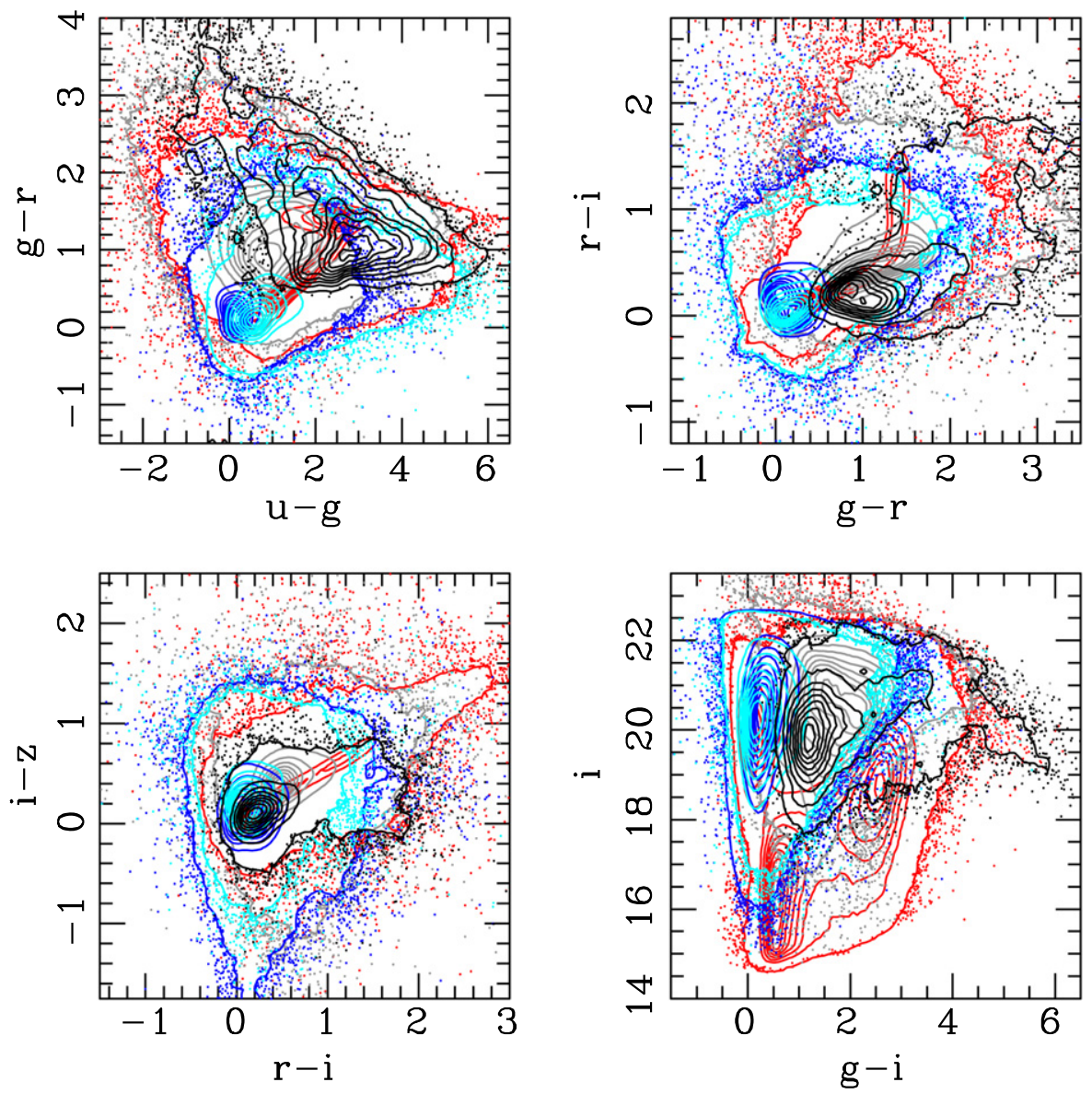

Figure 8. Optical colors of test set objects selected as quasar candidates. Contours/points and colors are as in Figure 5: high- $z$ quasars in black, mid- $z$ quasars in cyan, and low- $z$ quasars in blue. Training set "stars" are shown in red (for point sources) and gray (for extended sources).

$1.6 \%$, and $0.1 \%$ of the test set objects. These percentages are larger than expected from the priors; however, these include contaminants that we have worked to remove using some cuts as described below, and the algorithm is not strongly sensitive to differences at this level.

We have reduced the amount of contamination from stars and galaxies by restricting our analysis to objects classified as point sources in the SDSS photometry and by requiring that all the candidates lie to the right (redward) of both of the following two cuts:

$$
\begin{aligned}
& ([4.5] \leqslant 16.0 \& \&[3.6]-[4.5]<-0.1) \| \\
& ([4.5]>16.0 \& \&[3.6]-[4.5]<([4.5]-15.2) /-8.0)
\end{aligned}
$$

$$
\begin{aligned}
& (i \leqslant 16.5 \& \&[3.6]-[4.5]<-0.1) \| \\
& \quad(i>16.5 \& \&[3.6]-[4.5]<(i-15.7) /-8.0) .
\end{aligned}
$$

We further restrict our candidates to objects more than $15^{\circ}$ from the Galactic plane and that have less than 1 mag of extinction in the $u$ band, $A_{u}<1.0\left(A_{i}<0.4\right)$.

After these cuts we are left with 885,503 quasar candidates, including 748,839 low- $z$ candidates, 205,060 mid- $z$ candidates, and 13,060 high $z$ candidates, where the totals do not match owing to objects being selected in more than one redshift range. These numbers can be contrasted with the 5546 quasar candidates from our previous attempt at optical+MIR classification (Richards et al. 2009b). Four of the mid- $z$ objects and five of the low $-z$ objects are duplicates that result from matching of multiple IR sources to the same optical source; we have not resolved these duplicates into a single object in the interest of completeness.

Figures 8 and 9 mimic Figures 5 and 6, but here we plot the quasar candidates rather than the known quasars. Comparison of these distributions to the cuts used by Stern et al. (2012; solid black lines in Figure 9) and Assef et al. (2013; solid yellow lines in Figure 9) demonstrates the improvement of our method to type 1 quasars (particularly those that are faint with red optical colors) over using MIR color-magnitude cuts alone. While this vastly increases the number of high- $z$ quasar candidates, it does come at the cost of excluding type 2 quasar candidates.

\section{THE CATALOG}

Our catalog is presented in Table 2. Of the 885,503 quasar candidates, 733,713 lack spectroscopic confirmation (and 305,623 are objects that we have not previously classified as photometric quasar candidates). We find that 150,453 objects are already known to be quasars. This was determined by matching the candidates not only to the known quasars in the master quasar catalog that defined our training set but also to the full SDSS-I/II/III spectroscopic database. Only 743 candidates $(<0.1 \%)$ have been classified as stars. A total of 589 objects are classified as galaxies; however, 175 of those have $z>0.5$ and thus are likely to be AGNs. Indeed, many of 

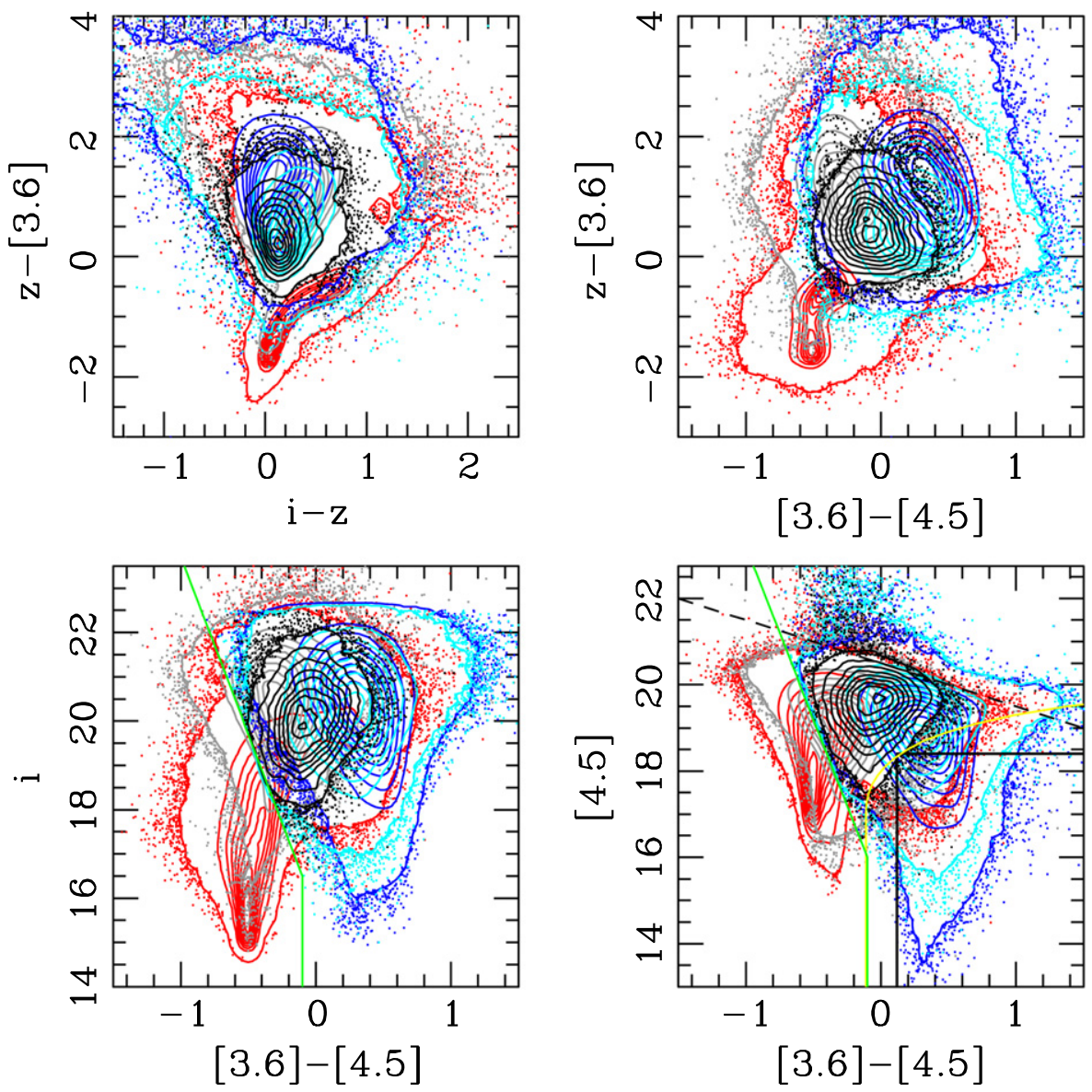

Figure 9. Optical colors of test set objects selected as quasar candidates. Contours/points and colors are as in Figures 6 and 8 . The dashed black line shows the detection limit as a function of color for a theoretical object with [3.6] $=20.5$. The solid black lines indicate the color and magnitude limits of the Stern et al. (2012) selection in AB magnitude space, while the yellow curve gives the 75\% reliability selection from Assef et al. (2013). The green lines in the bottom panels give our own cuts, as defined in Equations (3) and (4), which are intended to reduce stellar contamination.

the objects classified as $z>0.5$ galaxies appear in the handvetted SDSS quasar catalogs; this reflects the sensitivity of our method to low-luminosity AGNs in compact galaxies. The confirmed stars and galaxies do not occupy any unique parameter space that would allow them to be easily distinguished as contaminants. Overall, the candidate list appears to be quite robust, and visual inspection of the optical SDSS images confirms this impression.

The columns in the catalog are as follows: (1) R.A. (degrees; J2000); (2) decl. (degrees; J2000); (3) the classification of the object from matching to known objects (QSO, STAR, GALAXY, CELG, and "??") ${ }^{21}$ based on existing spectroscopy, or " $U$ " for unknown if we know of no spectroscopy for the source; and (4) the known redshift. Columns (5)-(11) give the ugriz optical $\mathrm{AB}$ (asinh) magnitudes (corrected for Galactic extinction) along with the [3.6] and [4.5] mid-IR $\mathrm{AB}$ magnitudes (also corrected for Galactic extinction). Columns (12)-(18) give the errors on these magnitudes. Columns (19)(32) give the SDSS-III, WISE, and Spitzer flux densities and errors, where the optical values are measured in nanomaggies (as reported by the SDSS data sweeps file that we use) and the mid-IR values have been converted to $\mu \mathrm{Jy}$; no extinction correction is applied to these values. Columns (33)-(40) give the YJHK magnitudes and errors from the UKIDSS or VHS

\footnotetext{
${ }^{21}$ See Section 5.3 for an explanation of the "CELG" and "??" classifications.
}

surveys (where available). Columns (41)-(44) give the far-UV (FUV) and near-UV (NUV) magnitudes and errors from GALEX (where available); no Galactic extinction corrections have been applied. Column (45) indicates whether the $g-i$ color is within $1 \sigma(0.68), 2 \sigma(0.95)$, or $3 \sigma(0.99)$ of the mean color for quasars at the predicted redshift. Outliers are an indication of either bad photometric redshifts or nonquasar contaminants. Column (46) is the $u$-band extinction from Schlafly \& Finkbeiner (2011); extinctions in other wavebands can be derived from this value. Columns (47) and (48) are the star and quasar probabilities as determined by the kernel density estimation used in our primary selection criterion. Columns (49)-(52) use the optical and MIR photometry to tabulate the minimum, best, and maximum photometric redshift along with the probability of being between the minimum and maximum values, as described in more detail in Section 4.1. Columns (53)-(56) are the same photometric redshift values but now adding JHK photometry to the optical and MIR. Column (57) indicates whether the object is within the "legacy" SDSS footprint, which is useful for statistical analysis. Column (58) indicates whether the object was in the uniform targeting area for the quasar target selection algorithm described in Richards et al. (2002). In Column (59) we give the flag (if set) from SDSS-DR7 quasar targeting, where Richards et al. (2002) and Schneider et al. (2010) provide details on the values of these flags - which can be used as a secondary indicator of 
Table 2

Optical+MIR Photometric Quasar Catalog

\begin{tabular}{|c|c|c|}
\hline Column & Name & Description \\
\hline 1 & R.A. & Right ascension (J2000) \\
\hline 2 & Decl. & Declination (J2000) \\
\hline 3 & CLASS & $\begin{array}{l}\text { Spectral classifcation (QSO, GALAXY, } \\
\text { STAR, CELG, ??, or U) }\end{array}$ \\
\hline 4 & ZSPEC & Spectroscopic redshift (if known) \\
\hline 5 & U_MAG & $\begin{array}{l}\text { SDSS } u \text {-band AB magnitude, corrected } \\
\text { for Galactic extinction }\end{array}$ \\
\hline 6 & G_MAG & $\begin{array}{l}\text { SDSS } g \text {-band AB magnitude, corrected } \\
\text { for Galactic extinction }\end{array}$ \\
\hline 7 & R_MAG & $\begin{array}{l}\text { SDSS } r \text {-band AB magnitude, corrected } \\
\text { for Galactic extinction }\end{array}$ \\
\hline 8 & I_MAG & $\begin{array}{l}\text { SDSS } i \text {-band AB magnitude, corrected for } \\
\text { Galactic extinction }\end{array}$ \\
\hline 9 & Z_MAG & $\begin{array}{l}\text { SDSS } z \text {-band AB magnitude, corrected } \\
\text { for Galactic extinction }\end{array}$ \\
\hline 10 & CH1_MAG & $\begin{array}{l}3.6 \mu \mathrm{m} \mathrm{AB} \text { magnitude, corrected for } \\
\text { Galactic extinction }\end{array}$ \\
\hline 11 & CH2_MAG & $\begin{array}{l}\text { 4.5 } \mu \mathrm{m} \mathrm{AB} \text { magnitude, corrected for } \\
\text { Galactic extinction }\end{array}$ \\
\hline 12 & U_MAG_ERR & Error on $u$-band magnitude \\
\hline 13 & G_MAG_ERR & Error on $g$-band magnitude \\
\hline 14 & R_MAG_ERR & Error on $r$-band magnitude \\
\hline 15 & I_MAG_ERR & Error on $i$-band magnitude \\
\hline 16 & Z_MAG_ERR & Error on $z$-band magnitude \\
\hline 17 & CH1_MAG_ERR & Error on $3.6 \mu \mathrm{m}$ magnitude \\
\hline 18 & CH2_MAG_ERR & Error on $4.5 \mu \mathrm{m}$ magnitude \\
\hline 19 & U_FLUX & $\begin{array}{l}\text { SDSS } u \text {-band flux density in } \\
\text { nanomaggies }\end{array}$ \\
\hline 20 & G_FLUX & $\begin{array}{l}\text { SDSS } g \text {-band flux density in } \\
\text { nanomaggies }\end{array}$ \\
\hline 21 & R_FLUX & SDSS $r$-band flux density in nanomaggies \\
\hline 22 & I_FLUX & SDSS $i$-band flux density in nanomaggies \\
\hline 23 & Z_FLUX & SDSS $z$-band flux density in nanomaggies \\
\hline 24 & CH1_FLUX & $3.6 \mu \mathrm{m}$ flux density in microJy \\
\hline 25 & CH2_FLUX & $4.5 \mu \mathrm{m}$ flux density in microJy \\
\hline 26 & U_FLUX_ERR & Error in $u$-band flux density \\
\hline 27 & G_FLUX_ERR & Error in $g$-band flux density \\
\hline 28 & R_FLUX_ERR & Error in $r$-band flux density \\
\hline 29 & I_FLUX_ERR & Error in $i$-band flux density \\
\hline 30 & Z_FLUX_ERR & Error in $z$-band flux density \\
\hline 31 & CH1_FLUX_ERR & Error in $3.6 \mu \mathrm{m}$ flux density \\
\hline 32 & CH2_FLUX_ERR & Error in $4.5 \mu \mathrm{m}$ flux density \\
\hline 33 & YAPERMAG3 & $\begin{array}{l}Y \text {-band Vega magnitude from UKIDSS } \\
\text { or VHS }\end{array}$ \\
\hline 34 & JAPERMAG3 & $\begin{array}{l}J \text {-band Vega magnitude from UKIDSS } \\
\text { or VHS }\end{array}$ \\
\hline 35 & HAPERMAG3 & $\begin{array}{l}H \text {-band Vega magnitude from UKIDSS } \\
\text { or VHS }\end{array}$ \\
\hline 36 & KSAPERMAG3 & $\begin{array}{l}K \text {-band Vega magnitude from UKIDSS } \\
\text { or VHS }\end{array}$ \\
\hline 37 & YAPERMAG3ERR & Error in $Y$-band magnitude \\
\hline 38 & JAPERMAG3ERR & Error in $J$-band magnitude \\
\hline 39 & HAPERMAG3ERR & Error in $H$-band magnitude \\
\hline 40 & KSAPERMAG3ERR & Error in $K$-band magnitude \\
\hline 41 & FUV_MAG & GALEX FUV magnitude (AB) \\
\hline 42 & FUV_MAG_ERR & GALEX NUV magnitude (AB) \\
\hline 43 & NUV_MAG & Error in FUV magnitude \\
\hline 44 & NUV_MAG_ERR & Error in NUV magnitude \\
\hline 45 & GI_SIGMA & $\begin{array}{l}\text { Indicator of distance from mean } g-i \\
\text { color at ZHOTBEST }\end{array}$ \\
\hline 46 & EXTINCTU & Extinction in SDSS $u$ band \\
\hline 47 & STAR_DENS & Star density from KDE algorithm \\
\hline 48 & QSO_DENS & Quasar density from KDE algorithm \\
\hline 49 & ZPHOTMIN & Minimum photometric redshift (ugriz) \\
\hline
\end{tabular}

Table 2

(Continued)

\begin{tabular}{|c|c|c|}
\hline Column & Name & Description \\
\hline 50 & ZPHOTBEST & Best photometric redshift (ugriz) \\
\hline 51 & ZPHOTMAX & Maximum photometric redshift (ugriz) \\
\hline 52 & ZPHOTPROB & $\begin{array}{l}\text { Probability of ZPHOTBEST being } \\
\text { between min and max }\end{array}$ \\
\hline 53 & ZPHOTMINJHK & $\begin{array}{l}\text { Minimum photometric redshift } \\
\quad(\text { ugrizJHK) }\end{array}$ \\
\hline 54 & ZPHOTBESTJHK & Best photometric redshift (ugrizJHK) \\
\hline 55 & ZРНOTMAXJHK & $\begin{array}{l}\text { Maximum photometric redshift } \\
\quad(\text { ugrizJHK) }\end{array}$ \\
\hline 56 & ZPHOTPROBJHK & $\begin{array}{l}\text { Probability of ZPHOTBESTJHK being } \\
\text { between min and max }\end{array}$ \\
\hline 57 & LEGACY & $\begin{array}{l}\text { Indicates if object is in the SDSS Legacy } \\
\text { footprint }\end{array}$ \\
\hline 58 & SDSS_UNIFORM & $\begin{array}{l}\text { Indicates if object was selected according } \\
\text { to Richards et al. (2002) }\end{array}$ \\
\hline 59 & PRIMTARGET & $\begin{array}{l}\text { SDSS primary target selection flag; see } \\
\text { Richards et al. (2002) }\end{array}$ \\
\hline 60 & PM & Proper motion in milliarcseconds per year \\
\hline 61 & DUPBIT & $\begin{array}{l}\text { Bitwise flag indicating low- } z\left(2^{0}\right) \text {, mid- } z \\
\left(2^{1}\right) \text {, and high } z\left(2^{2}\right) \text { sources }\end{array}$ \\
\hline
\end{tabular}

(This table is available in its entirety in FITS format.)

quasar likelihood. Column (60) gives the proper motion (PM) in mas per year in a similar manner as discussed in Richards et al. (2009a), based on Munn et al. (2004), and can also be used as a secondary indicator of quasar likelihood. Finally, column (61) is a bit-wise flag that indicates whether the object was selected as a low- $z$ (DUPBIT \& $2^{0}$ ), mid- $z$ (DUPBIT \& $2^{1}$ ), or high- $z$ (DUPBIT \& $2^{2}$ ) source (or a combination thereof).

\subsection{Photometric Redshifts}

We have used the photometric-redshift algorithm described by Richards et al. (2001) and Weinstein et al. (2004), extending it to include the mid-IR photometry from Spitzer and WISE and, in some cases, the near-IR photometry from VHS and UKIDSS. In short, this algorithm seeks to minimize the distance between the colors of an unknown source and the mean colors as shown in Figure 4. For luminous quasars this method is superior to template fitting (e.g., Assef et al. 2010, Figure 10) as it primarily picks up on the high-equivalent-width emission-line features rather than spectral breaks (although at high $z$ the Ly $\alpha$ break leads to improved photometric redshifts even with our method). Careful selection of templates can lead to improved results as shown by Salvato et al. (2009), particularly for host-dominated AGNs.

Figure 10 shows the photometric versus spectroscopic redshifts for all three samples. Note that there is some overlap between the samples (as designed to ensure that objects with redshifts near the edges of the training set redshift windows are not lost). The left panel reveals where there are photometric redshift degeneracies in the sample; however, the right panel shows that the vast majority have well-estimated photometric redshifts and that catastrophic outliers are a minority. We find that $90.9 \%, 82.7 \%$, and $85.7 \%$ of known quasars have photometric redshifts within $\delta z= \pm 0.3$, for high- $z$, mid- $z$, and low- $z$ candidates, respectively. Candidates can be restricted to more robust photometric redshifts by making a cut on 

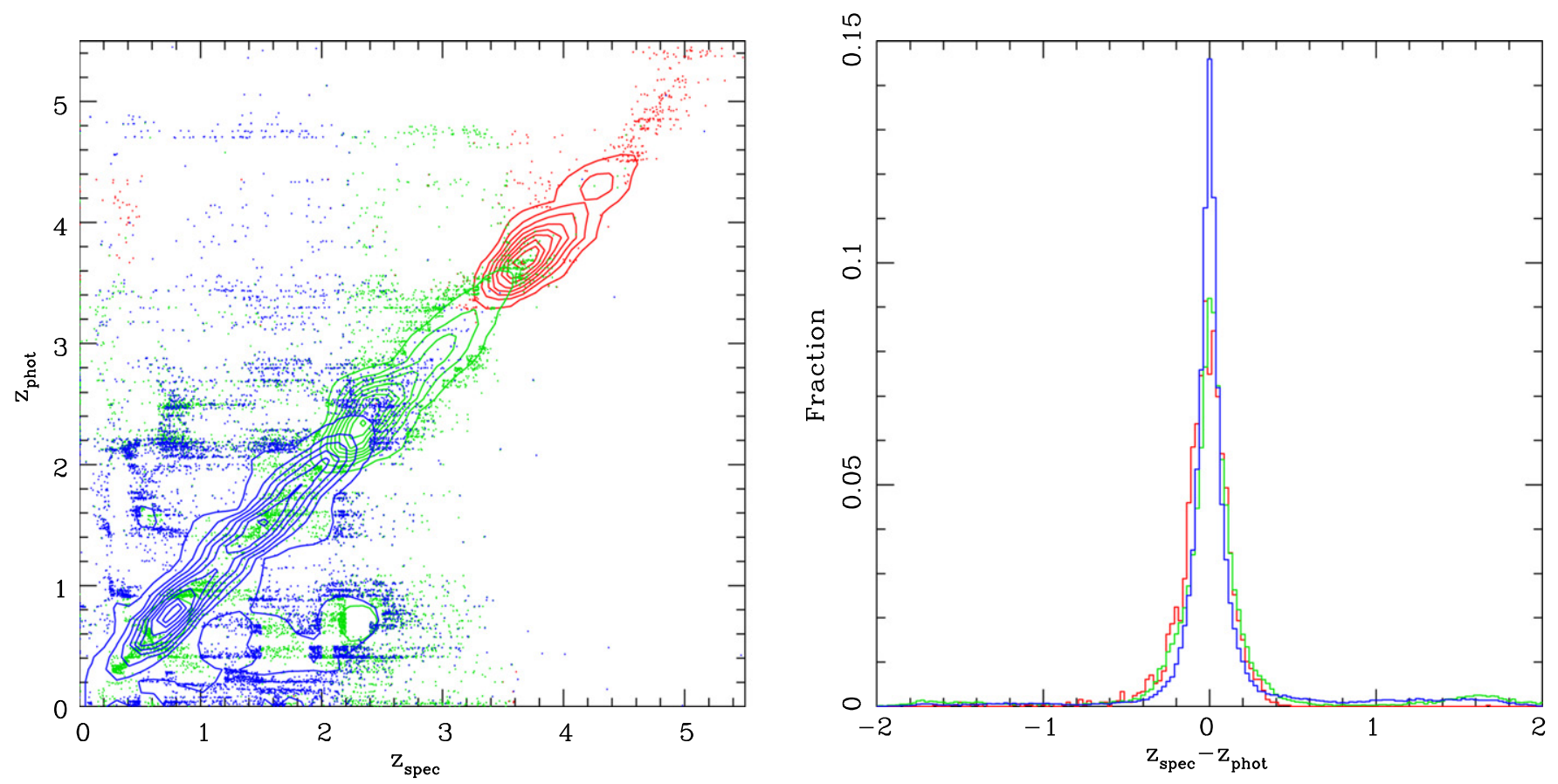

Figure 10. Left: photometric vs. spectroscopic redshift for all three samples; blue: low- $z$, green: mid- $z$, red: high- $z$. As this presentation highlights the catastrophic outliers at the expense of the well-determined photometric redshifts, we also present a histogram of the differences between the spectroscopic and photometric redshifts in the right panel. This shows that most objects have well-estimated redshifts.

ZPHOTPROB that gives the probability that the true redshift is between the minimum and maximum reported values.

It is not our goal herein to rigorously investigate the nature of the degeneracies in Figure 10. However, as one example, we consider the degeneracy between $z \sim 0.75$ and $z \sim 2.25$. Here the Ly $\alpha$ forest is not yet strong enough in $u$ to overcome similarities between the general optical/UV and MIR spectral slopes, $\mathrm{Mg}$ II versus $\mathrm{C}_{\text {IV }}$ in $g, \mathrm{H} \beta$ versus $\mathrm{Mg}_{\text {II }}$ in $z$, and $\mathrm{Pa} \alpha$ versus $\mathrm{Pa} \gamma$ in [3.6]. $J H K$ data can break that degeneracy as $J-K$ spans the $1 \mu$ m transition between the optical and IR at low redshift while it samples the optical slope at high redshift. We specifically find that adding $J H K$ data improves the overall photo- $z$ accuracy to $93 \%$ (virtually eliminating catastrophic errors). However, near-IR data of sufficient depth are only available over a fraction of the area surveyed; Euclid data (Laureijs et al. 2012) will be very welcome in this regard.

Another way we can determine the photometric redshift accuracy is to look at the color-redshift relation using the photometric redshifts of our objects. Figure 11 shows the distribution of $g-i$ color versus photometric redshift for our candidates. Photometric redshift degeneracies can produce semi-discrete features where one redshift is preferentially selected. Objects where the $g-i$ color is within the $99 \%$ confidence limit at the best-fit photometric redshift (GI_SIGMA) are highlighted in gray. These objects are likely to be the most robust candidates and are expected to have the most accurate photometric redshifts. Objects outside of this 99\% confidence interval are likely to be contaminants, have erroneous photometric redshifts, or have interesting spectral features (highly dust reddened, broad absorption lines, etc.). For example, the objects with colors bluer than the mean $g-i$ color at photometric redshifts of $z \sim 4.8$ and $z \sim 5.5$ are unlikely to be at those redshifts. However, they may well be quasars at $z \sim 4-4.5$. Alternatively, if they are indeed quasars, they could be at much lower redshift but have dust reddening or

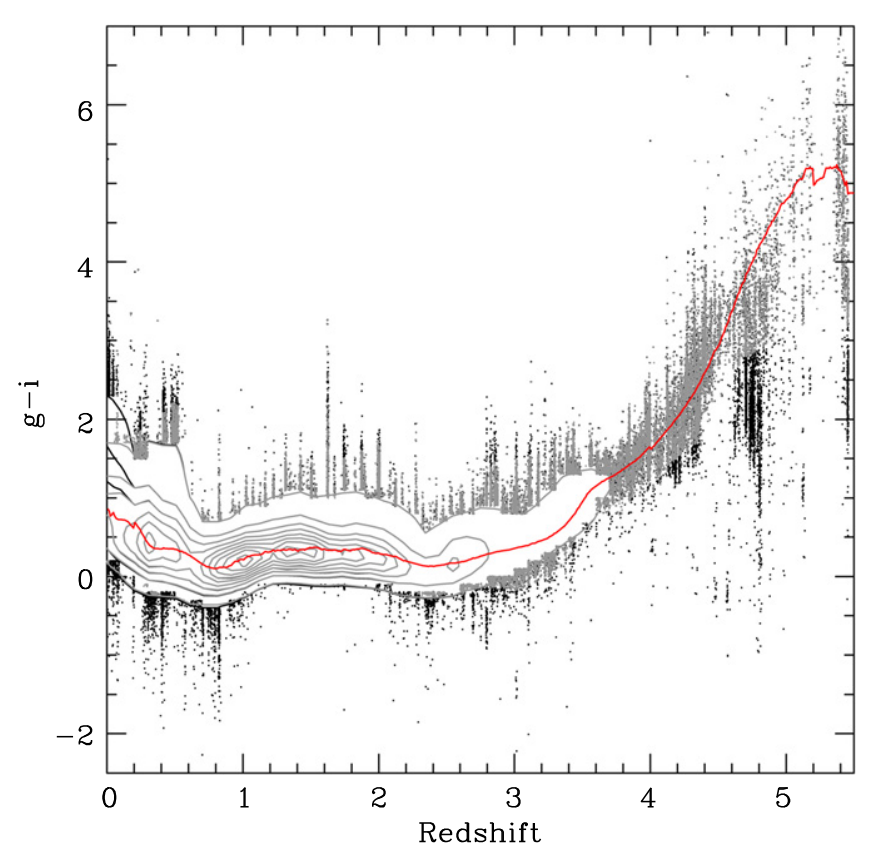

Figure 11. $g-i$ color vs. photometric redshift for our quasar candidates. Black (linear) contours and dots are all the candidates; gray contours and dots represent objects that have colors that are within the 99\% confidence limits of the mean quasar color-redshift relation (red line). Outliers may be contaminants or have erroneous photometric redshifts.

absorption troughs that make them appear like higher-redshift quasars.

\section{ANALYSIS}

\subsection{Comparison of Selection Methods}

An advantage of our selection method is that it can take full advantage of data from Spitzer during the post-cryogen 
exploration phase of the mission. In such cases, we only have 3.6 and $4.5 \mu \mathrm{m}$ measurements. This keeps us from being able to perform "wedge" selection that has proven so successful (Lacy et al. 2004; Stern et al. 2005, 2007) because WISE is not deep enough in $W_{3}$ and $W_{4}$ relative to our optical data. However, our method allows us to probe to much fainter IR limits using only two bands since we also have matched optical photometry. This process enables us to improve on MIR-only selection (Stern et al. 2012; Assef et al. 2013; at least within the SDSS footprint) by helping to remove the MIR bias against $3.5<z<5.0$ quasars. In a similar vein, our method is potentially more complete and more efficient at faint magnitudes than variability selection (e.g., Butler \& Bloom 2011), where the optical photometry in any single epoch of imaging is noisy. While the power-law method used by Donley et al. (2012) results in more reliable MIR classification, quasars are not necessarily power laws in the MIR (and are not always monotonic), so that method is more incomplete than that presented herein with regard to those objects that do not fit a power-law template (to within the errors).

We note that the Bovy et al. (2011) algorithm should perform similarly well to ours if it were rebuilt to include the stellar locus in the MIR (as opposed to applying color cuts before/after running the optical selection algorithm). One utility of the Bovy et al. (2011) method is that meaningful probabilities can be easily and rapidly built on a per-object basis. This allows for the alternative approach of constructing a fully probabilistic or extremely complete catalog, which is a less appropriate catalog to use for direct statistical analyses but which can be used to, e.g., match low-probability objects in the optical+MIR to AGN selected at other wavelengths (see DiPompeo et al. 2015, for just such a catalog). Alternatively, the catalog we have built is deliberately efficient (or "pure") and therefore more appropriate for statistical analyses given good characterization of the incompleteness.

An obvious question is what our method has gained over making simple color cuts. We illustrate this with two examples of MIR-only cuts and a cut involving both optical and MIR data. Figure 10 of Richards et al. (2009a) illustrates the tradeoff between completeness and contamination for a simple [3.6] - [4.5] color cut. The standard $W_{1}-W_{2}>0.8$ cut, which equates to [3.6] $-[4.5]>0.119$ as discussed above, would recover $80 \%$ of the quasar candidates compiled herein, with most of the losses being high-redshift candidates. The total number of test set objects passing such a cut is 1.85 million. If all of our candidates were quasars and all of the remaining objects within those 1.85 million were contaminants, then the contamination of such a cut would be $60 \%$. Restricting just to point sources leaves only 1 million targets, but that still would represent a contamination of $30 \%$. Thus, such a cut would be neither optimally complete nor efficient. If we wanted a more complete quasar set, a better cut would be [3.6] $-[4.5]>-0.1$, which achieves $95 \%$ completeness to our quasar candidates. However, it obviously comes with significantly greater contamination: $86 \%$ overall and $55 \%$ for point sources.

Better yet would be to combine the optical and MIR color information as we have in our KDE selection. A number of combinations are possible, but a simple cut of $i-[4.5]>(g-i)-1$ recovers $99 \%$ of our candidates. With that comes nearly $95 \%$ contamination as more than 18 million other objects are also selected by this cut. Most of that contamination is from normal galaxies, as restricting to point sources reduces the contamination to $50 \%$. A more restrictive cut to reduce the contamination is possible, but not without a commensurate reduction in completeness.

\subsection{Creating Robust Subsamples}

In order to further compare our candidates and selection algorithm to others, it is helpful to first identify the most robust subsamples possible. To that end, we consider the effects of star-galaxy separation, previous SDSS targeting flags, proper motion, and the presence of GALEX detections.

Particularly at high $z$, the robustness of our candidates depends on SDSS star-galaxy separation (as we might expect high- $z$ quasars to be point sources). The morphological classification is thought to be $95 \%$ correct at $r \sim 21$ (Annis et al. 2014), where this has been explored in more detail by Scranton et al. (2002). Figure 1 of Scranton et al. (2002) shows that, as signal-to-noise ratio degrades, galaxies are more likely to have small concentration indices and thus be classified as stars. "Point" sources fainter than 22nd mag have a significant probability of being galaxies; in poor seeing it is closer to $21 \mathrm{st}$ mag. As such, we do not consider any $i>22$ sources to be robust high- $z$ candidates (in the absence of other confirming information), and sources with $21<i<22$ deserve some caution.

In the case of relatively bright sources, the Richards et al. (2002) SDSS quasar target selection flags can be used to identify candidates that are particularly likely (or unlikely). As such, we have included those target flags (in the field PRIMTARGET) for sources where the SDSS-DR7 flag value was nonzero. Objects flagged as QSO_FAINT (PRIMTARGET \& 0x02000000) are sources that otherwise met the SDSS-DR7 selection criteria, but were just below the flux limit for spectroscopic follow-up. On the other hand, objects flagged as QSO_REJECT (PRIMTARGET \& 0x20000000) are in regions of color space known for high contamination. Based on the known quasars and the color cuts that defined this flag, objects with this flag set that do not have $z_{\text {phot }} \sim 2.4$ are likely to be less robust candidates.

In Richards et al. (2009a) we were able to remove some contaminants by identifying objects with high proper motions (Munn et al. 2004), and we have included the proper motion for those objects with quality proper motion measurements (having small errors and at least six epochs of data; see the discussion in Richards et al. 2009a). Using the same cuts as Richards et al. (2009a) removes 160 known quasars, which is just $0.25 \%$ of the quasars with quality proper motion measurements, yet it cuts 59 of the $280(21.1 \%)$ known stars. These criteria further cut 478 unknown objects $(0.73 \%)$, as compared to the 163 expected if all of those objects were quasars. Overall, we find that many fewer objects have large proper motion than in Richards et al. (2009a), which we attribute to the current catalog being less contaminated by stars.

We have not used UV data from GALEX in our selection or photometric redshift analysis, but we have further matched our catalog to GALEX data in order to identify contaminants and redshift errors. Specifically, we matched our candidate quasars to both the MIS and AIS GALEX catalogs as compiled by Bianchi et al. (2005), excluding sources with an NUV artifact flag. We then tabulate the NUV and FUV magnitudes (AB) in addition to their errors. This matching can be used to weed out 
low $z$ interlopers from among the high- $z$ candidates. Specifically, real high- $z$ quasars are relatively unlikely to be $G A L E X$ sources (particularly fainter sources). Alternatively, lowerredshift sources that we have misclassified as high- $z$ quasar candidates are much more likely to be detected by GALEX in the UV. We find that 101 of 9283 (1.1\%) known quasars in our sample with $z_{\text {spec }}>3$ are detected by GALEX, as compared to 313 of the 9547 objects $(3.3 \%)$ with $z_{\text {phot }}>3$, but that have low probability $(<0.8)$ of being at $z>3$. Thus, a GALEX detection for a high- $z$ candidate suggests that the candidate may not be robust.

The end result of these investigations is the addition of a number of parameters to our catalog that can be used to identify the most robust candidates. For our purposes, we will formally define "robust" candidates as those having ZPHOTPROB $>0.8$ and $a b s$ (GI_SIGMA) $<=0.95$. There are 517,586 candidates satisfying these criteria. Of those, only $717(0.14 \%)$ are known nonquasars, whereas 114,120 are known quasars.

For high- $z$ candidates $(3.5<z<5)$ we further restrict the most robust sources to nondetections in GALEX and $i<22$. There are 10,955 such sources, of which 7874 are unknown; 6779 of these have not been previously identified by us as photometric quasar candidates. Only 79 are nonquasar contaminants, while 2890 of the 3002 known quasars (96\%) indeed have $z>3$.

\subsection{COSMOS and Bootes}

One way to judge the utility of this catalog is to compare it to areas for which there is particularly dense spectroscopy. One such example is the COSMOS field (Sanders et al. 2007). In addition to the COSMOS spectroscopy discussed in Section 2.1, we also compared to Prescott et al. (2006), which further identifies objects in the COSMOS field. We recover 75 of the 95 quasars cataloged by them. Thirteen of these 75 were not identified as quasars in the master catalog, and we have updated their classifications in our catalog. Only three of our objects match to galaxies from Prescott et al. (2006), while no objects matched to stars.

This comparison suggests that our catalog is relatively complete to known COSMOS quasars and has relatively little contamination. Yet our catalog has nearly as many new quasar candidates within the COSMOS field as have been confirmed by spectroscopy. Within the area bounded by the COSMOS Spitzer data, we find 547 quasar candidates in total. Of these, 266 are known quasars, 3 are known galaxies, 1 is a known star, 32 are known compact emission-line galaxies (CELGs), 5 have spectra that are difficult to classify (given as "??" in the catalog), and 240 are unknown. CELG is a designation that we have chosen for those objects that are classified as narrow line in the COSMOS spectroscopy but generally show signs of being star-forming galaxies rather than being AGN powered. They are all fainter than $i=21$ and likely come into the sample as a result of a breakdown of SDSS star-galaxy separation as noted above. Of the unknown objects, only 95 are robust candidates as described above (20 with $z_{\text {phot }}>3.5$ and $i<22$ ). The lower-quality candidates have $i \sim 22$ and are at the limit of our selection method. Of the known quasars, only 5 have $z>3.5$ and 47 have $2.2 \leqslant z \leqslant 3.5$.

We can further compare our candidates to X-ray sources in the COSMOS field. The 2011 November update of the 53-field XMM-Newton data table analyzed in Brusa et al. (2010) contains 2000 X-ray sources. There are 264 matches (to within $\left.1^{\prime \prime}\right)$ to our catalog, 28 of which are unknown (16 robust). However, there are 176 additional unknown candidates (64 robust) from our catalog without X-ray matches that we deem within the X-ray footprint by virtue of there being an X-ray source within $240^{\prime \prime}$ (i.e., they are quasar candidates but were not detected in the X-ray). Of the robust candidates, 17 are $z>3.5$ candidates with $i<22$. Comparing the candidates matched to X-ray sources and those not matched, we find that the average $i$-band magnitude of the matches is 20.62, while for the nonmatches it is 21.96 . In terms of photometric redshift, the $\mathrm{X}$-ray matches have a mean value of 1.28 as compared to 2.88 for the nonmatches.

Chandra data in COSMOS cover a slightly smaller region. Using the Chandra Source Catalog, ${ }^{22}$ we find 934 X-ray sources, of which 125 match to our candidates, with 3 of those being objects without existing spectroscopy. However, there are another 125 of our quasars candidates within this X-ray footprint. Twenty of those are robust unknown sources, with 7 that are $z>3.5$ candidates with $i<22$ (all of which are included in the $X M M$ matching above). The average magnitude for these X-ray matches is $i=20.75$ and for the nonmatches is $i=21.54$. The mean photometric redshift for matches is $z=1.24$ and for nonmatches is 2.69 .

In principle, we could use morphology to further test the likelihood of the quasar classification of our candidates. However, the SDSS star-galaxy separation becomes unreliable at a brighter limit than our candidates. Although deep Hubble Space Telescope (HST) data are available in the COSMOS area (Scoville et al. 2007a), it is not definitive. While the known bright quasars do tend to have point-like morphologies, the faint quasars (even at high z) can be extended (host dominated) at the depth of the HST data. That said, any follow-up spectroscopy of COSMOS candidates should clearly consider the HST data for prioritization, as 6 of the 12 new high- $z$ candidates have stellar morphologies from HST (with the five nonmatches to the HST data all being near the edges of the COSMOS field).

If even a fraction of our mid- and high- $z$ quasar candidates in the COSMOS area are real quasars, it would significantly increase the number of such objects. Compared to only 5 known $z>3.5$ quasars among our candidates, we saw above that there are 17 robust $z>3.5$ candidates just within the X-ray footprint of the field, 6 of which have stellar HST morphologies - suggesting that the existing density of relatively bright high- $z$ quasars in the COSMOS field is at least $\sim 50 \%$ incomplete.

The photometric redshifts for COSMOS sources presented by Salvato et al. (2011) should be superior to ours and can be used to cross-check our results. However, only 36 of our candidates match: 12 mid- $z$ and just 1 high- $z$, likely because of the restriction to X-ray sources in Salvato et al. (2011). Of these, 17 have photometric redshifts that agree with ours to within \pm 0.3 ( 9 to \pm 0.1 ), including the high- $z$ candidate (COSMOS ID: 1980473), with a photometric redshift of 3.295 versus 3.329.

Brusa et al. (2009) report $40 z>3$ quasars in the COSMOS field (22 spectroscopic, 18 photometric). We recover only 7 of those (all of which already appear in the master quasar catalog); however, this is not surprising, as 32 of the 33 missing have $i>20.5$ (the peak of our distribution), and 27 have $i>22$;

\footnotetext{
22 http://cxc.harvard.edu/csc/
} 
thus, the Brusa et al. (2009) objects are much fainter than those cataloged herein.

As with the COSMOS field, the Boötes field has also been subject to considerable spectroscopic exploration, primarily from the AGES program (Kochanek et al. 2012). Within a rectangular area defined by the minimum and maximum R.A. and decl. of the deep Spitzer data taken as part of the SDWFS (Ashby et al. 2009), we find 1861 quasar candidates. Among these are 1085 confirmed quasars, 2 stars, and 3 galaxies, leaving 771 unknown objects. However, the Spitzer data do not fully cover this space: there are 1738 candidates (of which 681 have no spectroscopic data) that are included within the approximate boundaries of the MIR data. Some of those objects fall outside of the boundaries of the AGES spectroscopic program (Kochanek et al. 2012, Figure 2), but nevertheless have the deep MIR data needed to perform robust MIR selection.

Matching back to the AGES spectroscopy (to recover nonquasars not included in the training set), we find an additional 3 spectroscopically confirmed stars and 36 spectra that resulted in unknown redshift/classification. A search of the NASA Extragalactic Database for additional spectroscopic data revealed only one new object, FBQS J142607.7+340426, that was not included in our master quasar catalog.

Thus, as with the COSMOS field, the Boötes field also contains many new quasar candidates, despite considerable efforts to confirm likely AGNs. Of the 771 unknown candidates, we find that 294 are robust, with 46 being robust $z>3.5$ candidates with $i<22$.

As a result of this analysis of quasar candidates in the COSMOS and Boötes fields, we conclude that there is a potential for significantly increasing the number of relatively bright high- $z$ (and mid- $z$ ) quasars in that area of sky-despite considerable existing spectroscopic coverage of the field. The density of objects in these (and other Spitzer deep fields) is particularly useful for absorption studies, making additional confirming spectroscopy worthwhile.

\subsection{Demographics}

One of our goals was to fill in the gaps at redshifts where optical-only quasar selection has traditionally been incomplete. The SDSS selection algorithm (Richards et al. 2002) targets both low-redshift and high-redshift quasars to $i<19.1$. To that limit the SDSS quasar sample is expected to be quite complete at $z<2.2$, with known incompleteness at $z \sim 2.7$ and $z \sim 3.5$ (Vanden Berk et al. 2005; Richards et al. 2006; Worseck \& Prochaska 2011). Similarly, the BOSS selection algorithm (Ross et al. 2012a) is limited to $\sim 2.2<z<\sim 3.5$ and has known incompleteness at $z \sim 2.9$ (Ross et al. 2012b). We would thus expect to find that our method would have little new to offer in terms of new bright quasars $(i<19.1)$ at $z<2.2$, but may significantly improve quasar selection around $z \sim 2.7$ and $z \sim 3.5$. We might expect somewhat more new quasar candidates between $19.1<i<20.2$ as SDSS did not target quasars at $z<3$ fainter than $i=19.1$ (reserving the fainter targets for $z>3$ candidates - targeted to $i<20.2$ ) and BOSS did not explicitly target $z<2.2$ quasars.

In this light, we have matched our candidate list to the full master catalog (to determine which of these objects are new candidates), to the training sets (to determine the completeness with respect to the quasar training set), and to the full SDSS-III spectroscopic database (to identify known nonquasars).

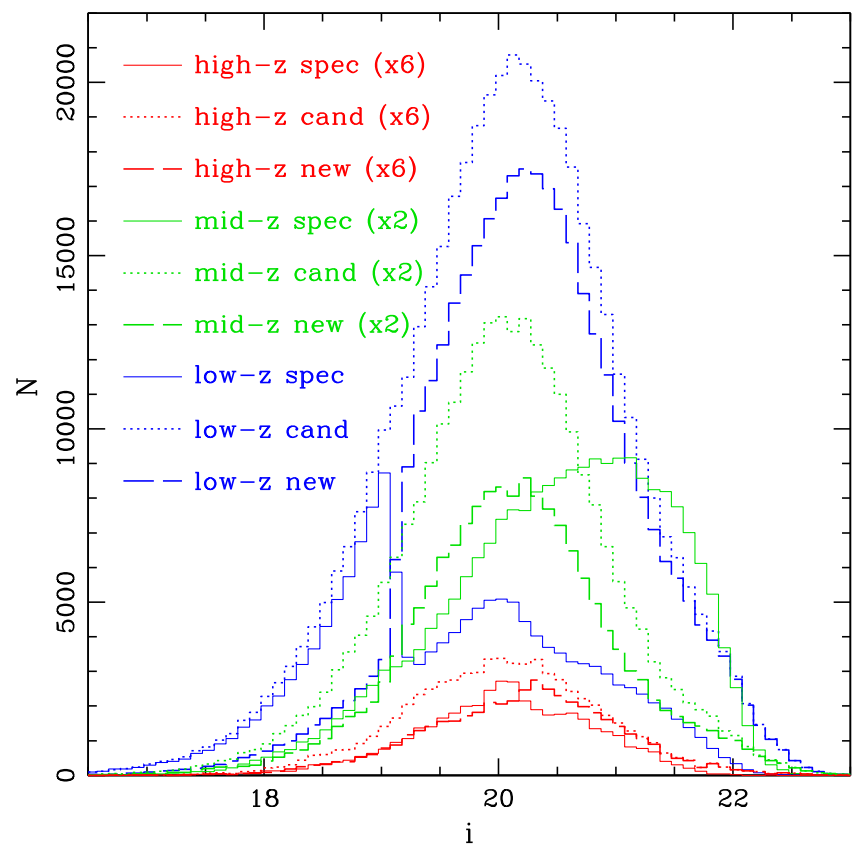

Figure 12. Number counts of known quasars and robust quasar candidates as a function of magnitude and redshift range. Blue lines show the number of known quasars with $z<2.2$ ("spec"; thin), the number of low-z-selected candidates ("cand"; dotted), and the number of low-z-selected candidates that lack spectroscopic confirmation ("new"; dashed). Similarly, green and red lines give the number of $2.2<z<3.5$ (or mid-z-selected) and $3.5<z<5.5$ (or high- $z$-selected) quasars and quasar candidates. The green curves are scaled up by a factor of 2 and the red curves are scaled up by a factor of 6 in order to make the figure more legible.

Figure 12 compares the number of known spectroscopic quasars, our robust quasar candidates, and those robust candidates without existing spectroscopy. Comparing the low- $z$ quasars/candidates (blue lines), we find that there are some new quasars at $i<19.1$ (the SDSS spectroscopic limit for $z \lesssim 3$ ), which may reflect our sensitivity to low-luminosity AGNs in compact galaxies. There are also hundreds of thousands of new low- $z$ objects at fainter magnitudes.

For mid- $z$-selected quasars $(2.2<z<3.5)$, Figure 12 shows that our catalog provides relatively little in terms of new sources at $i<19.1$ and $i>21 .^{23}$ However, at intermediate magnitudes, the number of new candidate mid- $z$ quasars is quite substantial. In some sense this is surprising as the SDSSIII BOSS project was specifically designed to find quasars in this magnitude and redshift range. At the same time, it is known that BOSS is only $\sim 60 \%$ complete (Ross et al. 2012b), so it is quite possible that we are simply turning up the remaining objects missed by BOSS. For high-z-selected quasars $(z>3.5)$, we again find relatively few new objects brighter than the SDSS spectroscopic limit (here $i<20.2$ ), but there is a significant population of new candidates at fainter magnitudes-consistent with the difficulty that standard wedge-based IR selection of AGNs (Lacy et al. 2004; Stern et al. 2005, 2007) has to recover objects at these redshifts.

The expected redshift distribution of the robust new candidates is shown in Figure 13. We have computed the ratio

\footnotetext{
${ }^{23}$ Note that the thin solid lines in Figure 12 show the total number of known spectroscopic quasars, not the number of such objects that also have mid-IR photometry. Thus, even if the candidate object number counts are below the spectroscopic counts, that does not necessarily indicate that we are incomplete to known quasars with MIR data.
} 


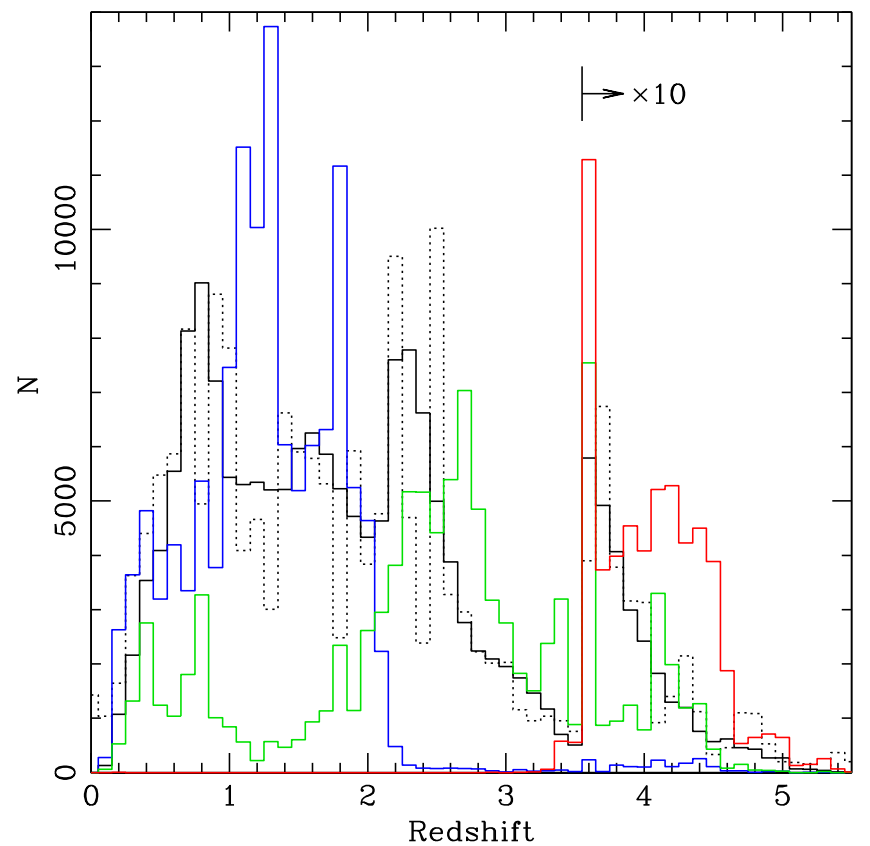

Figure 13. Number of quasars as a function of redshift. The solid black line gives the spectroscopic redshift distribution of the quasars in our training set, while the dotted black line gives the photometric redshift distribution for those same sources. The ratio of these two is used to perform a first-order correction of the photometric redshift distribution of our candidates. Corrected photometric redshift distributions for the robust new candidates (spectroscopically confirmed sources removed) are shown in blue for low- $z$ candidates (scaled down by $3 \times$ to fit on the axis), green for mid- $z$, and red for high- $z$. All histograms are scaled up by $10 \times$ for $z>3.6$ to better show the high-redshift distribution.

of the photometric and spectroscopic redshift distributions for the spectroscopically confirmed quasars in our training set. This enables a rough correction of the photometric redshift distributions of our candidates to an expected spectroscopic redshift distribution (shown in blue, green, and red for low- $z$, mid- $z$, and high $z$ candidates, respectively). As noted above, the low- $z$ candidates are largely faint sources; they generally have photometric redshifts consistent with their low- $z$ selection. The mid- $z$ candidates have a large range of photometric redshifts, which suggests photo- $z$ degeneracy and/or contamination. There are a large number of mid- $z$ candidates with photometric redshifts of $z \sim 2.7$ and $z \sim 3.5$, which is encouraging as these are redshift regions where we know that optical-only selection is incomplete (Richards et al. 2002, 2006). The high- $z$ candidates all have redshift estimates consistent with their selection, with a large number of new objects spanning $3.6<z<4.6$

We find that most of the new candidates are at fainter magnitudes and/or come at redshifts where it is difficult to do optical-only, variability-only, or infrared-only selection of quasars. For example, many new candidates are at high redshift, which tend to be biased against by traditional mid-IR selection methods, as noted above, and also by variability selection methods. Overall, there are 7874 robust high- $z$ quasar candidates. If all turned out to be quasars, this would more than double the number of such quasars in the SDSS footprint. Many of these candidates are very faint, but the distribution peaks at $i \sim 20.5$, likely reflecting the cutoff of $i=20.2$ for high- $z$ quasar selection in SDSS-I/II. In the mid- $z$ range there are 81,321 robust quasar candidates. At low $z$ there are 424,448

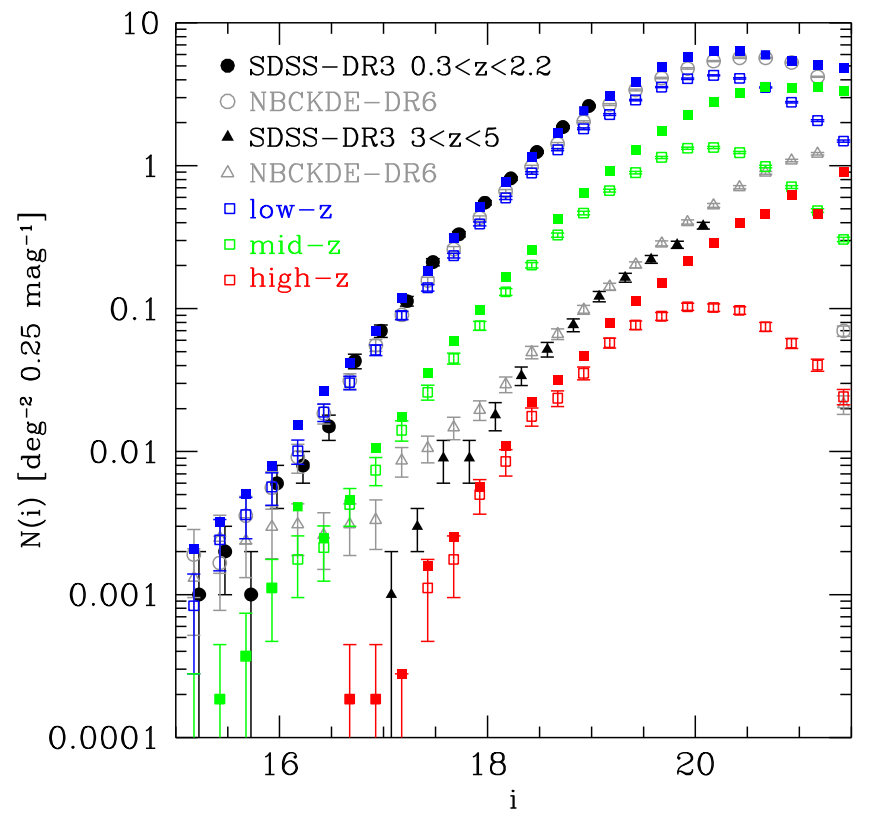

Figure 14. Quasar number counts as a function of redshift and $i$-band magnitude. Black and gray points, respectively, give the spectroscopic and photometric number counts as reported in (e.g., Figure 9 of Richards et al. 2009a); circles for $z<2.2$ and triangles for $3<z<5$. The open blue, green, and red squares give the raw number counts (with $1 \sigma$ Poisson error bars) for the candidates reported herein. The filled colored squares give the number counts corrected using Figure 15. The mid- $z$ and high $z$ samples bracket the redshift space of the old $3<z<5$ sample, but show no sign of the contamination at the bright end (flattening of the number counts) seen in the old sample.

robust quasar candidates. Most of these are quite faint, and despite the catalog's limitation to point sources, those with $z_{\text {phot }}<1$ are likely AGNs rather than luminous quasars.

Many of these candidates are identified in our previous photometric quasar catalogs. However, a total of 87,242, 34,059, and 6779 low- $z$, mid- $z$, and high- $z$ candidates, respectively, do not already appear in Richards et al. (2009a) or Bovy et al. (2011).

\subsection{Number Counts/Luminosity Function}

A particularly useful test for a sample of photometric quasars is a comparison of their number counts to those of known quasars. Problems with efficiency/contamination will show up as an excess of quasars (particularly at bright magnitudes), while problems with completeness will show up as a dearth of quasars.

In Figure 14 we reproduce Figure 9 from Richards et al. (2009a), which showed both the spectroscopic and photometric quasar number counts in two redshift ranges. Here we have overplotted the number counts of our quasar candidates selected as low- $z$, mid- $z$, and high- $z$ candidates.

In this figure, open points represent the raw number counts, while the filled points give the completeness-corrected number counts. As we will do for the luminosity function analysis below, the objects going into the raw number counts presented here are limited to those in the SDSS "legacy area" (area $=10778.306 \mathrm{deg}^{2}$ ) and are classified as either quasars or unknown. The unknown sources are restricted to robust candidates as defined above in Section 5.2. The completeness corrections for this sample are given by the fraction of master quasar catalog objects recovered by our algorithm with these 


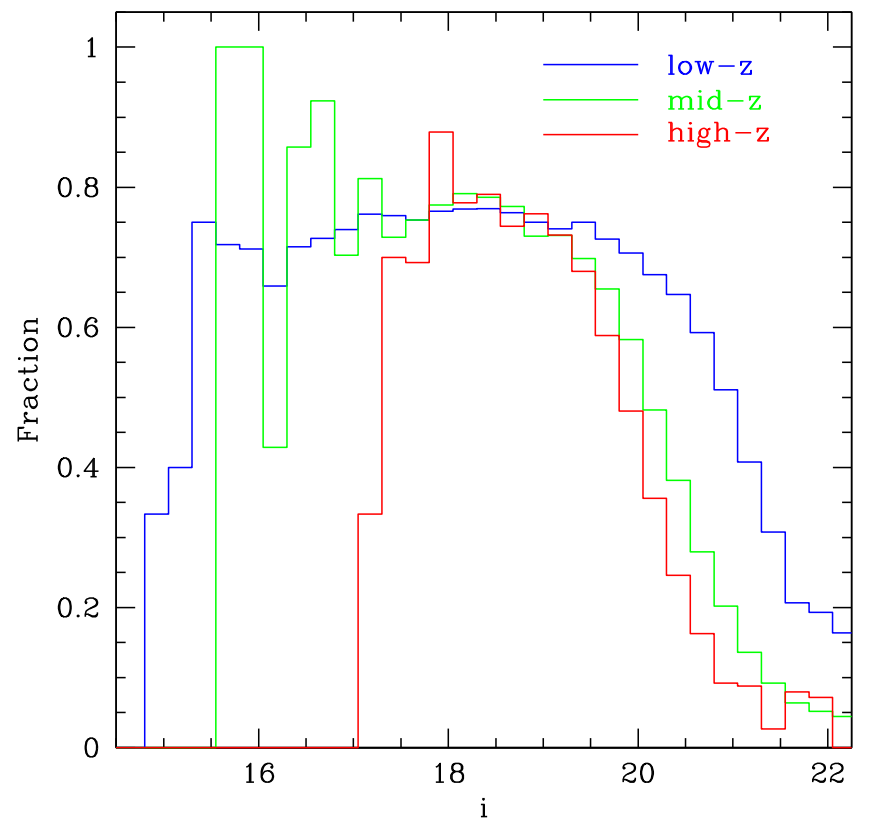

Figure 15. Ratio of objects in the master catalog objects recovered by our algorithm to the master quasar catalog (prior to matching to mid-IR photometry). This corrects for objects too faint in the mid-IR to match the optical, objects rejected from the IR catalog due to flags, the exclusion of extended sources, and the incompleteness of the selection algorithm itself. The fraction is given as a function of $i$-band magnitude in the three redshift ranges we have explored (low-z: blue; mid- $z$ : green; high- $z$ : red).

constraints, as shown in Figure 15. This analysis converts the raw counts to the total number of quasars expected (accounting for incompleteness of the selection algorithm, lack of mid-IR photometry, nonstellar morphology, and flag rejection).

We specifically find that the corrected low- $z$ number counts are a good match to the spectroscopic number counts at $i \sim 17$, being somewhat incomplete at $i \sim 19$ (but probing to $i \sim 20.5$ ), and exhibiting perhaps a factor of two contamination in the brightest bin shown. For mid- $z$ quasars our sample appears to be filling in the gap in the SDSS selection over $19.1<i<20.2$, while exhibiting less contamination than our previous photometric sample (as evidenced by a lack of a plateau at the bright end). The high- $z$ number counts do not show any obvious sign of contamination from bright stars (once we have imposed the restrictions noted above).

These number counts are thus consistent with our new catalog being both relatively complete (to within a deterministic correction) and efficient. If the efficiency was low (and thus the contamination was high), we would expect significant deviations from the slopes of the spectroscopic number counts. We see none of the excess in our current photometric sample that we saw in the high- $z$ sample from Richards et al. (2009a), and the faint-end counts are consistent with the optical +infrared selected candidates from Figure 12 of Richards et al. (2009b), which performed a selection similar to our current selection, but over a much smaller area of sky $\left(\sim 24 \mathrm{deg}^{2}\right)$.

While our goal in this work was not to determine the luminosity function of quasars, but rather to take the next step in creating optimal photometric catalogs of quasars, it is nevertheless useful to examine the QLF as determined from our catalog. In Figure 16 we show the absolute magnitude (luminosity) and photometric redshift distribution of our data

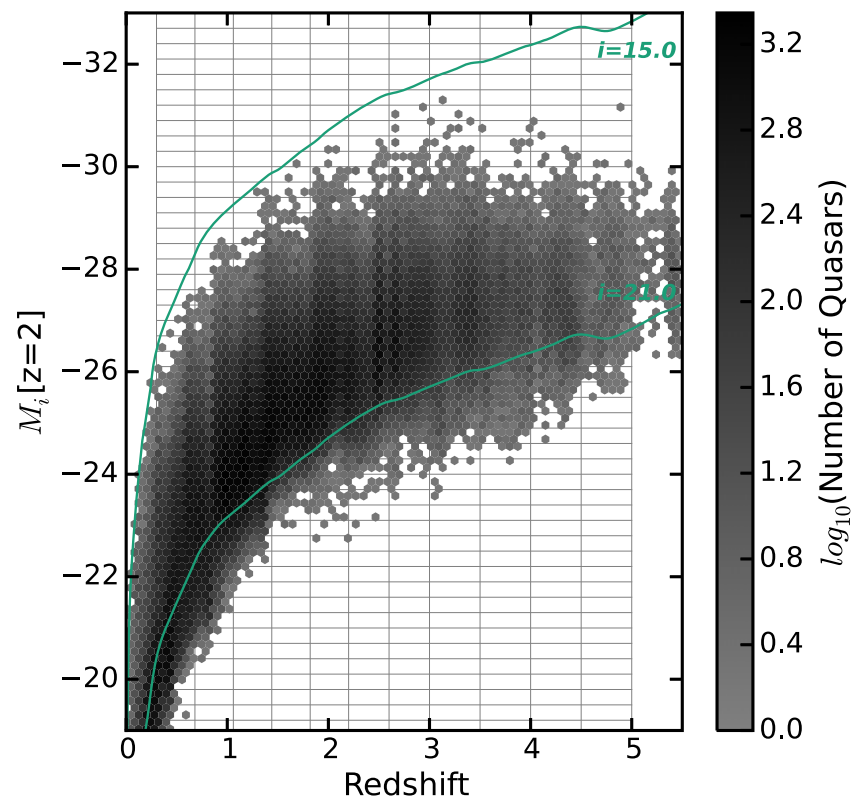

Figure 16. Absolute magnitude (luminosity) vs. redshift for our photometric quasar candidates with number of objects displayed as grayscale hex bins. Teal lines indicate the bright limit of SDSS and the adopted limiting magnitude of our QLF analysis. The light gray grid lines delineate the bins used to compute the QLF in Figure 17.

using the same redshift and luminosity bins as Richards et al. (2006), and we compare the resulting luminosity function in these bins in Figure 17. We have taken the limiting magnitude to be $i<21$ as shown, since that is where our completeness falls below $50 \%$ according to Figure 3 . However, there is no single limiting magnitude for this investigation as we have simply matched all of the SDSS optical sources with MIR sources from WISE and Spitzer. Objects fainter than $i=21$ can be included in the catalog if they are bright enough in the MIR, but they are excluded from our main QLF analysis. The gradient in the density of points near the $i=21$ limit in Figure 16 might suggest that we are complete to deeper than this limit at low $z$, but also that the completeness is at a somewhat brighter magnitude at high $z$.

To produce the QLF results shown in Figure 17, we restricted the catalog using the same cuts as above for the number counts, namely, limiting to known quasars and "robust" unknown sources, both within the legacy area. In this presentation we make two corrections to the raw data. First, we correct for incompleteness as a function of $i$-band magnitude and redshift by weighting by the fraction of training set quasars recovered by our algorithm. Next, we correct the photometric redshifts by weighting each object by the ratio of the number of spectroscopic redshifts to photometric redshifts for our training set quasars. That is, if there were really 100 spectroscopic training-set quasars at $z=1.45-1.55$, but the photometric redshift estimates for those quasars placed 120 quasars in the same bin, then we would weight each new photometric quasar candidate in that photo- $z$ bin by $100 / 120$.

We find reasonable agreement with the spectroscopic QLF points of Richards et al. (2006) given that the focus of this work was not the rigorous computation of the QLF. Specifically, the black points in Figure 17 are in good agreement with the SDSS points (gray) down to the flux limit of SDSS and appear to be well behaved another magnitude deeper than the SDSS data. 


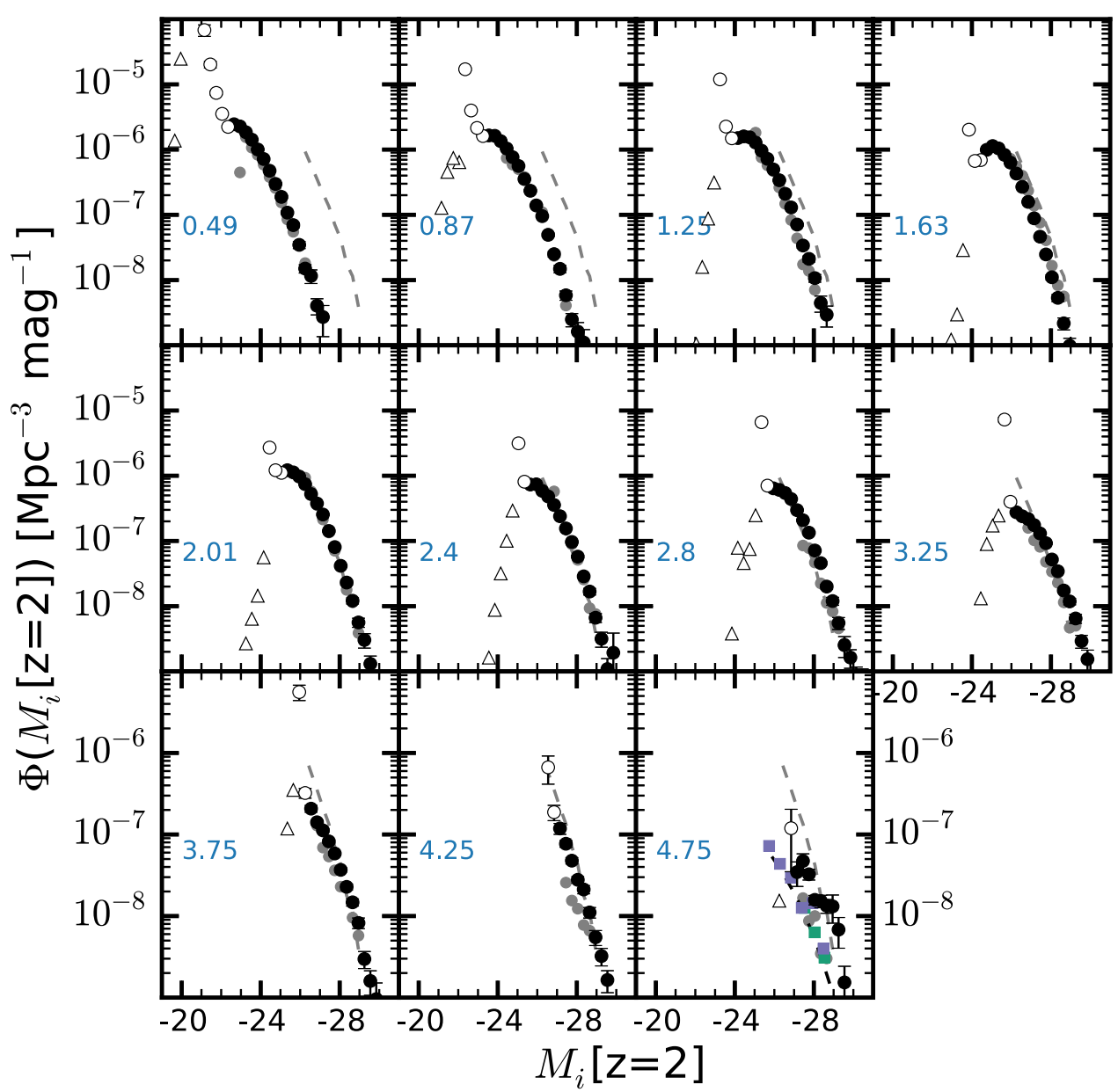

Figure 17. Quasar luminosity function in 11 redshift bins. Filled black circles are photometric objects from our catalogs brighter than the adopted limiting magnitude. Open circles are those where the limiting magnitude cuts though the $(L, z)$ bin and thus have uncertain corrections (error bars are Poisson only), while the open triangles indicate (uncorrected) lower limits. Gray points are at the spectroscopic QLF values from Richards et al. (2006), where the dashed gray line repeats the spectroscopic QLF from $z=2$ in each redshift panel. In the $z=4.75$ panel, we overplot the data (purple and teal) and best fit (dashed black line) from McGreer et al. (2013). The photometric QLF matches the spectroscopic QLF quite well, especially considering that this was not one of the goals of this investigation. The excess density at $z=4.25$ indicates either an undercorrection of the completeness by Richards et al. (2006) or contamination in our sample-likely a combination of both.

An exception is the deviation from Richards et al. (2006) seen in the $z=4.25$ panel, where our photometric sample has a space density that is a factor of a few higher than SDSS at $M_{i}(z=2) \sim-27$. This is likely to be caused by either contamination from nonquasars in our sample or undercorrection of the SDSS completeness in this redshift range. If it is incompleteness, the origin may be a greater sensitivity of our method to dust-reddened (but unobscured) quasars. Indeed, Lacy et al. (2015), using an MIR-selected sample, similarly find a high fraction of redder quasars at high redshift. Interestingly, this high-redshift QLF exhibits a steeper QLF slope than Richards et al. (2006) and is more consistent with the results of Jiang et al. (2008), Ross et al. (2012b), and McGreer et al. (2013). At $z=4.75$ the errors are somewhat larger, but the QLF is broadly consistent with McGreer et al. (2013).

\subsection{Future}

One of the goals of this work is to set the stage for nextgeneration clustering investigations using high-redshift quasars. The SDSS quasar sample lacks sufficient density to test the luminosity dependence of quasar clustering (Lidz et al. 2006) such as proposed by Hopkins et al. (2007). For example, the work by Shen et al. (2007) used a sample of only $\sim 4000$ quasars at $2.9<z<5.4$ over $\sim 4000 \mathrm{deg}^{2}$. Here we cover more than double that area and nearly double the sample size, but over an even smaller redshift range. The various optical and MIR deep fields would enable the discovery of more objects by probing much deeper, but they are limited in their utility for high- $z$ clustering investigations by their small area and the MIR bias against high- $z$ quasars.

Substantial gains should come from pairing this method with the data coming from the SpIES project (Timlin et al. 2015), which has just completed tiling $\sim 125 \mathrm{deg}^{2}$ of the SDSS Stripe 82 region (e.g., Annis et al. 2014). We can estimate the number of high $-z$ quasars in the SpIES area from the SWIRE ELAIS$\mathrm{N} 2$ field (4.2 $\left.\mathrm{deg}^{2}\right)$, which has the same depth as SpIES (but has not been covered by SERVS). In that field we find 32 high- $z$ quasar candidates, 24 of which appear to have robust photometric redshifts. Thus, we predict that SpIES will contain of order 5-7 high $-z$ quasars per square degree, or a total of $625-875$ objects. This density should be sufficient for powerful tests of the clustering of quasars as a function of luminosity at high redshift.

This work is further a proof of concept for future quasar surveys using both ground- and space-based data, such as could 
be done by combining photometric data from Pan-STARRS (Kaiser et al. 2002; grizy), SkyMapper (Keller et al. 2007; uvgriz), the DES (The Dark Energy Survey Collaboration 2005; grizY), Hyper Suprime-Cam ${ }^{24}$ (grizy), the LSST (Ivezic et al. 2008; ugrizy), the NEOWISE extension to the WISE program (Mainzer et al. 2014; using the two shortest WISE bandpasses), Euclid (Laureijs et al. 2012; YJH), or for future spectroscopic programs like the Dark Energy Spectroscopic Instrument (DESI; Schlegel et al. 2011). We have shown that using the combination of optical and MIR photometry is better (for unobscured quasars) than either data set alone and that there are considerable gains to be made from the use of modern statistical methods in performing multidimensional selection.

\section{CONCLUSIONS}

Using a proven kernel density estimation technique, we identify 885,503 type 1 quasar candidates within the imaging footprint of the Sloan Digital Sky Survey by combining the SDSS optical data with mid-IR imaging from WISE and Spitzer. Among these objects are 6779 robust, $3.5<z<5$ quasar candidates that have no previous spectroscopic or photometric classification. This increase is possible as a result of incompleteness of MIR-only color selection in this redshift range and the difficulty of variability selection for faint, highredshift quasars and offers an opportunity to expand our exploration of the high-redshift universe.

The optical and MIR color distributions shown in Figures 8 and 9 are good matches to the distributions of the training set quasars, but extend to fainter limits in both the optical and MIR. They also clearly demonstrate an increased completeness to high-redshift quasars (particularly at $3.5<z<5$, where MIR color selection is incomplete owing to spectral features pushing the colors of these objects bluer than typical MIR color cuts).

Photometric redshift estimates of these candidates using optical and MIR photometry are accurate to $\Delta z \pm 0.3$ at least $83 \%$ of the time, improving to $93 \%$ where there also exists near-IR photometry; see Figure 10. Comparison with the known colors of objects at the expected redshift (Figure 11) can help to identify potential contaminants and/or those objects with erroneous photo- $z$.

Our new candidates even include robust targets within the well-covered COSMOS and Boötes fields, where an increased density of spectroscopic quasars would aid in clustering and absorption-line studies. This includes over 50 robust, new high$z$ quasar candidates in both of the fields (where there exists deeper-than-average MIR photometry).

Generally, our algorithm is simply finding quasars that are fainter than the SDSS spectroscopic limits and that should not necessarily have received SDSS spectroscopic follow-up. However, there are a number of bright low $z$ candidates without SDSS spectroscopy that are likely to be low-luminosity AGNs rather than luminous quasars. Figures 12 and 13 present the magnitude and expected redshift distributions of both the new candidates and the known quasars.

We are able to explore the completeness and contamination of the method using number counts and luminosity function analysis. Figure 14 demonstrates that our algorithm is relatively complete to known low- $z$ quasars (accounting for our restriction to optical point sources) and shows no obvious sign

\footnotetext{
${ }^{24}$ http://www.naoj.org/Projects/HSC/surveyplan.html
}

of contamination from bright stars at any redshift. The QLF shown in Figure 17 agrees well with the results from SDSS (Richards et al. 2006) but suggests a steeper slope to the QLF at high $z$ (consistent with McGreer et al. 2013) and may be more sensitive to dust-reddened quasars. Future work will expand that presented herein by incorporating more information (variability, proper motion, etc.) and using survey data that probe deeper in the optical.

G.T.R. and A.D.M. acknowledge the generous support of a research fellowship from the Alexander von Humboldt Foundation at the Max-Planck-Institut für Astronomie, and G.T.R. is grateful for the hospitality of the Astronomisches RechenInstitut. This work was supported in part by NASA-ADAP grant NNX12AI49G and NSF grant 1411773. A.D.M. acknowledges support from NASA-ADAP grant NNX12AE38G and NSF grant 1211112. Funding for the SDSS and SDSS-II has been provided by the Alfred P. Sloan Foundation, the Participating Institutions, the National Science Foundation, the U.S. Department of Energy, the National Aeronautics and Space Administration, the Japanese Monbukagakusho, the Max Planck Society, and the Higher Education Funding Council for England. The SDSS is managed by the Astrophysical Research Consortium for the Participating Institutions. The Participating Institutions are the American Museum of Natural History, Astrophysical Institute Potsdam, University of Basel, Cambridge University, Case Western Reserve University, University of Chicago, Drexel University, Fermilab, the Institute for Advanced Study, the Japan Participation Group, Johns Hopkins University, the Joint Institute for Nuclear Astrophysics, the Kavli Institute for Particle Astrophysics and Cosmology, the Korean Scientist Group, the Chinese Academy of Sciences (LAMOST), Los Alamos National Laboratory, the Max-Planck-Institute for Astronomy (MPIA), the Max-Planck-Institute for Astrophysics (MPA), New Mexico State University, Ohio State University, University of Pittsburgh, University of Portsmouth, Princeton University, the United States Naval Observatory, and the University of Washington. Funding for SDSS-III has been provided by the Alfred P. Sloan Foundation, the Participating Institutions, the National Science Foundation, and the U.S. Department of Energy Office of Science. The SDSS-III Web site is http://www.sdss3.org/. SDSS-III is managed by the Astrophysical Research Consortium for the Participating Institutions of the SDSS-III Collaboration, including the University of Arizona, the Brazilian Participation Group, Brookhaven National Laboratory, Carnegie Mellon University, University of Florida, the French Participation Group, the German Participation Group, Harvard University, the Instituto de Astrofisica de Canarias, the Michigan State/Notre Dame/ JINA Participation Group, Johns Hopkins University, Lawrence Berkeley National Laboratory, Max Planck Institute for Astrophysics, Max Planck Institute for Extraterrestrial Physics, New Mexico State University, New York University, Ohio State University, Pennsylvania State University, University of Portsmouth, Princeton University, the Spanish Participation Group, University of Tokyo, University of Utah, Vanderbilt University, University of Virginia, University of Washington, and Yale University. This work is based (in part) on archival data obtained with the Spitzer Space Telescope, which is operated by the Jet Propulsion Laboratory, California Institute of Technology under a contract with NASA. Support for this 
work was provided by an award issued by JPL/Caltech (\#1378034). This publication makes use of data products from the Wide-field Infrared Survey Explorer, which is a joint project of the University of California, Los Angeles, and the Jet Propulsion Laboratory/California Institute of Technology, funded by the National Aeronautics and Space Administration. This work has made extensive use of the TOPCAT (Taylor et al. 2005) and STILTS (Taylor et al. 2006) tools. We thank Joe Hennawi, Nadia Zakamska, Alex Gray, and the anonymous referee for their contributions.

Facilities: Sloan, Spitzer, WISE.

\section{REFERENCES}

Ahn, C. P., Alexandroff, R., Allende Prieto, C., et al. 2012, ApJS, 203, 21 Aihara, H., Allende Prieto, C., An, D., et al. 2011, ApJS, 193, 29 Annis, J., Soares-Santos, M., Strauss, M. A., et al. 2014, ApJ, 794, 120 Ashby, M. L. N., et al. 2009, ApJ, 701, 428

Assef, R. J., Kochanek, C. S., Brodwin, M., et al. 2010, ApJ, 713, 970 Assef, R. J., Stern, D., Kochanek, C. S., et al. 2013, ApJ, 772, 26 Bertin, E., \& Arnouts, S. 1996, A\&AS, 117, 393

Bianchi, L., Seibert, M., Zheng, W., et al. 2005, ApJL, 619, L27 Blanton, M. R., Schlegel, D. J., Strauss, M. A., et al. 2005, AJ, 129, 2562 Bovy, J., et al. 2011, ApJ, 729, 141

Bovy, J., Myers, A. D., Hennawi, J. F., et al. 2012, ApJ, 749, 41 Brusa, M., Comastri, A., Gilli, R., et al. 2009, ApJ, 693, 8 Brusa, M., Civano, F., Comastri, A., et al. 2010, ApJ, 716, 348 Butler, N. R., \& Bloom, J. S. 2011, AJ, 141, 93

Cardelli, J. A., Clayton, G. C., \& Mathis, J. S. 1989, ApJ, 345, 245 Croom, S. M., Smith, R. J., Boyle, B. J., et al. 2004, MNRAS, 349, 1397 Croom, S. M., Richards, G. T., Shanks, T., et al. 2009, MNRAS, 392, 19 The Dark Energy Survey CollaborationarXiv:astro-ph/0510346 Dawson, K. S., Schlegel, D. J., Ahn, C. P., et al. 2013, AJ, 145, 10 DiPompeo, M. A., Bovy, J., Myers, A. D., \& Lang, D. 2015, MNRAS, 452, 3124

Donley, J. L., Koekemoer, A. M., Brusa, M., et al. 2012, ApJ, 748, 142 Eisenhardt, P. R., Stern, D., Brodwin, M., et al. 2004, ApJS, 154, 48 Eisenstein, D. J., Weinberg, D. H., Agol, E., et al. 2011, AJ, 142, 72 Fabricant, D., Fata, R., Roll, J., et al. 2005, PASP, 117, 1411 Fan, X., Strauss, M. A., Richards, G. T., et al. 2006, AJ, 131, 1203 Fazio, G. G., Hora, J. L., Allen, L. E., et al. 2004, ApJS, 154, 10 Fukugita, M., Ichikawa, T., Gunn, J. E., et al. 1996, AJ, 111, 1748 Giannantonio, T., Crittenden, R., Nichol, R., \& Ross, A. J. 2012, MNRAS, 426, 2581

Glikman, E., Bogosavljević, M., Djorgovski, S. G., et al. 2010, ApJ, 710, 1498 Gray, A. G., Richards, G., Nichol, R., Brunner, R., \& Moore, A. W. 2005 Proc. PhyStat 05, Statistical Problems in Particle Physics, Astrophysics and Cosmology (Singapore: World Scientific)

Gunn, J. E., Carr, M., Rockosi, C., et al. 1998, AJ, 116, 3040

Gunn, J. E., Siegmund, W. A., Mannery, E. J., et al. 2006, AJ, 131, 2332

Hennawi, J. F., Myers, A. D., Shen, Y., et al. 2010, ApJ, 719, 1672

Hewett, P. C., \& Wild, V. 2010, MNRAS, 405, 2302

Hinshaw, G., Larson, D., Komatsu, E., et al. 2013, ApJS, 208, 19

Hopkins, P. F., Lidz, A., Hernquist, L., et al. 2007, ApJ, 662, 110

Ivezic, Z., Tyson, J. A., Allsman, R., et al. 2008, arXiv:0805.2366

Jiang, L., Fan, X., Annis, J., et al. 2008, AJ, 135, 1057

Jiang, L., Fan, X., Bian, F., et al. 2014, ApJS, 213, 12

Kaiser, N., Aussel, H., Burke, B. E., et al. 2002, Proc. SPIE, 4836, 154

Keller, S. C., Schmidt, B. P., Bessell, M. S., et al. 2007, PASA, 24, 1

Kochanek, C. S., Eisenstein, D. J., Cool, R. J., et al. 2012, ApJS, 200, 8

Krawczyk, C. M., Richards, G. T., Mehta, S. S., et al. 2013, ApJS, 206, 4

Lacy, M., Ridgway, S. E., Sajina, A., et al. 2015, arXiv:1501.04118

Lacy, M., Storrie-Lombardi, L. J., Sajina, A., et al. 2004, ApJS, 154, 166

Lacy, M., Wilson, G., Masci, F., et al. 2005, ApJS, 161, 41

Laureijs, R., Gondoin, P., Duvet, L., et al. 2012, Proc. SPIE, 8442, 0

Lawrence, A., Warren, S. J., Almaini, O., et al. 2007, MNRAS, 379, 1599

Leistedt, B., \& Peiris, H. V. 2014, MNRAS, 444, 2

Leistedt, B., Peiris, H. V., Mortlock, D. J., Benoit-Lévy, A., \& Pontzen, A. 2013, MNRAS, 435, 1857

Lidz, A., Hopkins, P. F., Cox, T. J., Hernquist, L., \& Robertson, B. 2006, ApJ, 641,41
Lilly, S. J., le Fèvre, O., Renzini, A., et al. 2007, ApJS, 172, 70

Lonsdale, C. J., Smith, H. E., Rowan-Robinson, M., et al. 2003, PASP, 115,897

Lupton, R. H., Gunn, J. E., \& Szalay, A. S. 1999, AJ, 118, 1406

Maddox, N., Hewett, P. C., Péroux, C., Nestor, D. B., \& Wisotzki, L. 2012, MNRAS, 424, 2876

Mainzer, A., Bauer, J., Cutri, R. M., et al. 2014, ApJ, 792, 30

Mauduit, J.-C., Lacy, M., Farrah, D., et al. 2012, PASP, 124, 1135

McGreer, I. D., Jiang, L., Fan, X., et al. 2013, ApJ, 768, 105

McMahon, R. 2012, Science from the Next Generation Imaging and Spectroscopic Surveys (Garching: ESO)

Ménard, B., Scranton, R., Fukugita, M., \& Richards, G. 2010, MNRAS, 405, 1025

Messias, H., Afonso, J., Salvato, M., Mobasher, B., \& Hopkins, A. M. 2012 , ApJ, 754, 120

Munn, J. A., Monet, D. G., Levine, S. E., et al. 2004, AJ, 127, 3034

Myers, A. D., Brunner, R. J., Richards, G. T., et al. 2006, ApJ, 638, 622

Oguri, M., Inada, N., Pindor, B., et al. 2006, AJ, 132, 999

Papovich, C., Cool, R., Eisenstein, D., et al. 2006, AJ, 132, 231

Pâris, I., Petitjean, P., Aubourg, É, et al. 2012, A\&A, 548, A66

Pâris, I., Petitjean, P., Aubourg, É, et al. 2014, A\&A, 563, A54

Prescott, M. K. M., Impey, C. D., Cool, R. J., \& Scoville, N. Z. 2006, ApJ, 644, 100

Pullen, A. R., \& Hirata, C. M. 2013, PASP, 125, 705

Richards, G. T., Weinstein, M. A., Schneider, D. P., et al. 2001, AJ, 122,1151

Richards, G. T., Fan, X., Newberg, H. J., et al. 2002, AJ, 123, 2945

Richards, G. T., Nichol, R. C., Gray, A. G., et al. 2004, ApJS, 155, 257

Richards, G. T., Strauss, M. A., Fan, X., et al. 2006, AJ, 131, 2766

Richards, G. T., Myers, A. D., Gray, A. G., et al. 2009a, ApJS, 180, 67

Richards, G. T., Deo, R. P., Lacy, M., et al. 2009b, AJ, 137, 3884

Riegel, R., Gray, A. G., \& Richards, G. 2008, in Proc. SIAM Int. Conf. on Data Mining SDM 2008, ed. C. Apte et al. (Atlanta, GA: SIAM), 208

Ross, N. P., Myers, A. D., Sheldon, E. S., et al. 2012a, ApJS, 199, 3

Ross, N. P., McGreer, I. D., White, M., et al. 2012b, arXiv:1210.6389

Salvato, M., Hasinger, G., Ilbert, O., et al. 2009, ApJ, 690, 1250

Salvato, M., Ilbert, O., Hasinger, G., et al. 2011, ApJ, 742, 61

Sanders, D. B., Salvato, M., Aussel, H., et al. 2007a, ApJS, 172, 86

Sanders, D. B., Salvato, M., Aussel, H., et al. 2007b, ApJS, 172, 86

Schlafly, E. F., \& Finkbeiner, D. P. 2011, ApJ, 737, 103

Schlegel, D., Abdalla, F., Abraham, T., et al. 2011, arXiv:1106.1706

Schmidt, M., \& Green, R. F. 1983, ApJ, 269, 352

Schneider, D. P., Richards, G. T., Hall, P. B., et al. 2010, AJ, 139, 2360

Scoville, N., Abraham, R. G., Aussel, H., et al. 2007a, ApJS, 172, 38

Scoville, N., Aussel, H., Brusa, M., et al. 2007b, ApJS, 172, 1

Scranton, R., Johnston, D., Dodelson, S., et al. 2002, ApJ, 579, 48

Scranton, R., Ménard, B., Richards, G. T., et al. 2005, ApJ, 633, 589

Shen, Y., Strauss, M. A., Oguri, M., et al. 2007, AJ, 133, 2222

Skrutskie, M. F., Schneider, S. E., Stiening, R., et al. 1997, in ASSL 210, The Impact of Large Scale Near-IR Sky Surveys, (Berlin: Springer), 25

Stern, D., Eisenhardt, P., Gorjian, V., et al. 2005, ApJ, 631, 163

Stern, D., Kirkpatrick, J. D., Allen, L. E., et al. 2007, ApJ, 663, 677

Stern, D., Assef, R. J., Benford, D. J., et al. 2012, ApJ, 753, 30

Stoughton, C., Lupton, R. H., Bernardi, M., et al. 2002, AJ, 123, 485

Taylor, M. B. 2005, in ASP Conf. Ser. 347, Astronomical Data Analysis Software and Systems XIV, ed. P. Shopbell, M. Britton \& R. Ebert (San Francisco, CA: ASP), 29

Taylor, M. B. 2006, in ASP Conf. Ser. 351, Astronomical Data Analysis Software and Systems XV, ed. C. Gabriel et al. (San Francisco, CA: ASP), 666

Timlin, J., Ross, N., Richards, G. T., et al. 2015, in American Astronomical Society Meeting Abstracts, Vol. 225, American Astronomical Society Meeting Abstracts, 336.18

Trump, J. R., Impey, C. D., Elvis, M., et al. 2009, ApJ, 696, 1195

Vanden Berk, D. E., Schneider, D. P., Richards, G. T., et al. 2005, AJ, 129,2047

Weinstein, M. A., Richards, G. T., Schneider, D. P., et al. 2004, ApJS, 155,243

Worseck, G., \& Prochaska, J. X. 2011, ApJ, 728, 23

Wright, E. L., Eisenhardt, P. R. M., Mainzer, A. K., et al. 2010, AJ, 140,1868

Wu, X.-B., Hao, G., Jia, Z., Zhang, Y., \& Peng, N. 2012, AJ, 144, 49

Xue, Y. Q., Luo, B., Brandt, W. N., et al. 2011, ApJS, 195, 10

York, D. G., Adelman, J., Anderson, J. E., et al. 2000, AJ, 120, 1579 